\title{
Preparation and thermal stability of optically active 1,2,4-triazolium-based ionic liquids
}

\author{
Paweł Borowiecki, ${ }^{a^{*}}$ Marcin Poterała, ${ }^{\text {a Jan Maurin, }}$ \\ Monika Wielechowska ${ }^{a}$ and Jan Plenkiewicz ${ }^{a}$ \\ ${ }^{a}$ Warsaw University of Technology, Faculty of Chemistry, Noakowskiego St. 3, 00-664 \\ Warszawa, Poland, ${ }^{b}$ National Centre of Nuclear Research, 05-400 Otwock-Świerk, Poland \\ E-mail:pawel_borowiecki@onet.eu
}

\begin{abstract}
The synthesis of optically active ionic liquids, in a four-step reaction sequence, is described. In the first step an oxirane ring of cyclohexene oxide was opened with 1,2,4-triazole, yielding a racemic mixture of $(1 R, 2 R)$ - and $(1 S, 2 S)-2-(1 H-1,2,4-t r i a z o l-1-y l) c y c l o h e x a n o l$. Kinetic resolution of the racemate by a lipase catalyzed transesterification with vinyl acetate followed by alkylation (quaternization) of the triazole ring resulted in the appropriate optically active salts formation. After the anion metathesis, thermally stable novel chiral ionic liquids were obtained.
\end{abstract}

Keywords: Optically active ionic liquids, 1,2,4-triazolium salts, kinetic resolution

\section{Introduction}

The growing interest in recent years in chiral ionic liquids inclined us ${ }^{1}$ to prepare and investigate some properties of a few 1,2,4-triazolium-based salts. Ionic liquids (ILs) are salts consisting of big organic cations and inorganic or organic anions with melting points lower than $100{ }^{\circ} \mathrm{C}$ $(373.15 \mathrm{~K}){ }^{2}$ The existence of an enormous range of cation-anion combinations, ${ }^{3,4}$ gives lot of possibilities for modification of IL's structure resulting in diverse chemical and physical properties. By changing the cation or anion in an ionic liquid molecule, such property as density, melting point, viscosity, or solubility in water or other solvents - can be changed and fine-tuned. That is why ILs are often called 'designer solvents' or considered as 'task-specific' compounds, which fulfill and influence the outcome of technological demands in various applications. At present, ionic liquids are widely used as solvents in chemical synthesis ${ }^{5}$ as well as in electrochemistry ${ }^{6}$ and in reactions carried out with enzymes or microorganisms. ${ }^{7}$ Obviously, beside achiral ILs, their chiral, optically active analogues (CILs), became a subject of intensive study in recent years. As it was established, chiral ionic liquids can act as catalysts for asymmetric induction ${ }^{8}$ or as supplements influencing reaction stereoselectivity. ${ }^{9}$ They can also be used as a chiral solvents in stereoselective polymerization, ${ }^{10}$ as a chiral phase for gas 
chromatography ${ }^{11}$ or as chiral shift reagents in NMR. ${ }^{12}$ They were also applied in production of chiral liquid crystals. ${ }^{13}$ Beside the applications mentioned above, some ILs posses special and unique antibacterial or antifungal activities, which is promising for future development of new disinfectants, sanitizers, preservatives or highly toxic biocides. ${ }^{14}$ For example Pernak et al. ${ }^{15}$ obtained ionic liquids based on acesulfamate and choline, and tested them as insect feeding deterrents, fixatives for soft tissues in histopathological diagnosis and preservatives for blood.

It was found that quaternized nitrogen heterocycles in IL molecules give usually lower melting compounds than those containing aliphatic ammonium ions, and for this reason, mostly imidazolium or pyridinium cations were used in ionic liquids preparation. A few achiral ILs containing a quaternized triazole ring in the molecule are also described. Because the geometry and coordinating properties of 1,2,4-triazoles are similar to those of imidazoles, one can also expect they can provide interesting and valuable ionic liquids. Moreover, as it is known that the presence of a 1,2,4-triazole ring in a molecule often creates interesting pharmacological activities, many 1,2,4-triazole derivatives exhibit antibacterial, antifungal, anticancer, antitubercular, analgesic or anti-inflammatory properties. ${ }^{16}$ Unique physical properties of ILs in combination with their possible biological activity seemed to be very interesting, and inclined us ${ }^{1}$ to prepare a few new 1,2,4-triazolium based ionic liquids. A similar investigation was undertaken by Spanish researchers ${ }^{17}$ and some of the results are convergent, however the employed procedures and final products were different.

\section{Results and Discussion}

Herein, we describe a simple and efficient synthetic procedure for the preparation of optically active triazolium quaternary salts as potential ionic liquids. The salts were prepared from enantiomerically enriched $(1 S, 2 S)$-2-(1H-triazol-1-yl)cyclohexanol and (1R,2R)-2-(1H-triazol-1yl)cyclohexyl acetate obtained through lipase mediated kinetic resolution of the racemate. The starting racemic trans-( \pm$)-2-(1 H-1,2,4-t r i a z o l-1-y l) c y c l o h e x a n o l \quad( \pm)-3$, was synthesized according to the method described by Yus and co-workers ${ }^{18}$ by stirring equimolar amounts of 1,2,4-triazole (2) and cyclohexene oxide (1) without catalyst and in the absence of any solvent. We found that the solvent-free ring opening of cyclohexene oxide by 1,2,4-triazole is regioselective and the reaction yield can be improved by a slight increase of the temperature as well as the extension of the reaction time (Table 1).

Table 1. Ring opening of cyclohexene oxide (1) with 1,2,4-triazole (2) under solvent-free conditions

\begin{tabular}{ccccc}
\hline Entry & Product & Time, $\mathrm{h}$ & $T,{ }^{\circ} \mathrm{C}$ & Yield, $\%^{\mathrm{a}}$ \\
\hline 1 & $\mathbf{3}$ & 16 & 60 & 38 \\
2 & $\mathbf{3}$ & 12 & 65 & 42 \\
3 & $\mathbf{3}$ & 72 & 65 & 68 \\
\hline
\end{tabular}

${ }^{\text {a }}$ Isolated yield of pure product after recrystallization from $i$-PrOH. 
The racemic acetyl ester ( \pm -4 4 required for determination of conversion rates and enantiomeric excesses in all enzyme catalyzed reactions was prepared analogously to the method reported by Busto et al. ${ }^{19}$ as shown in Scheme 1.

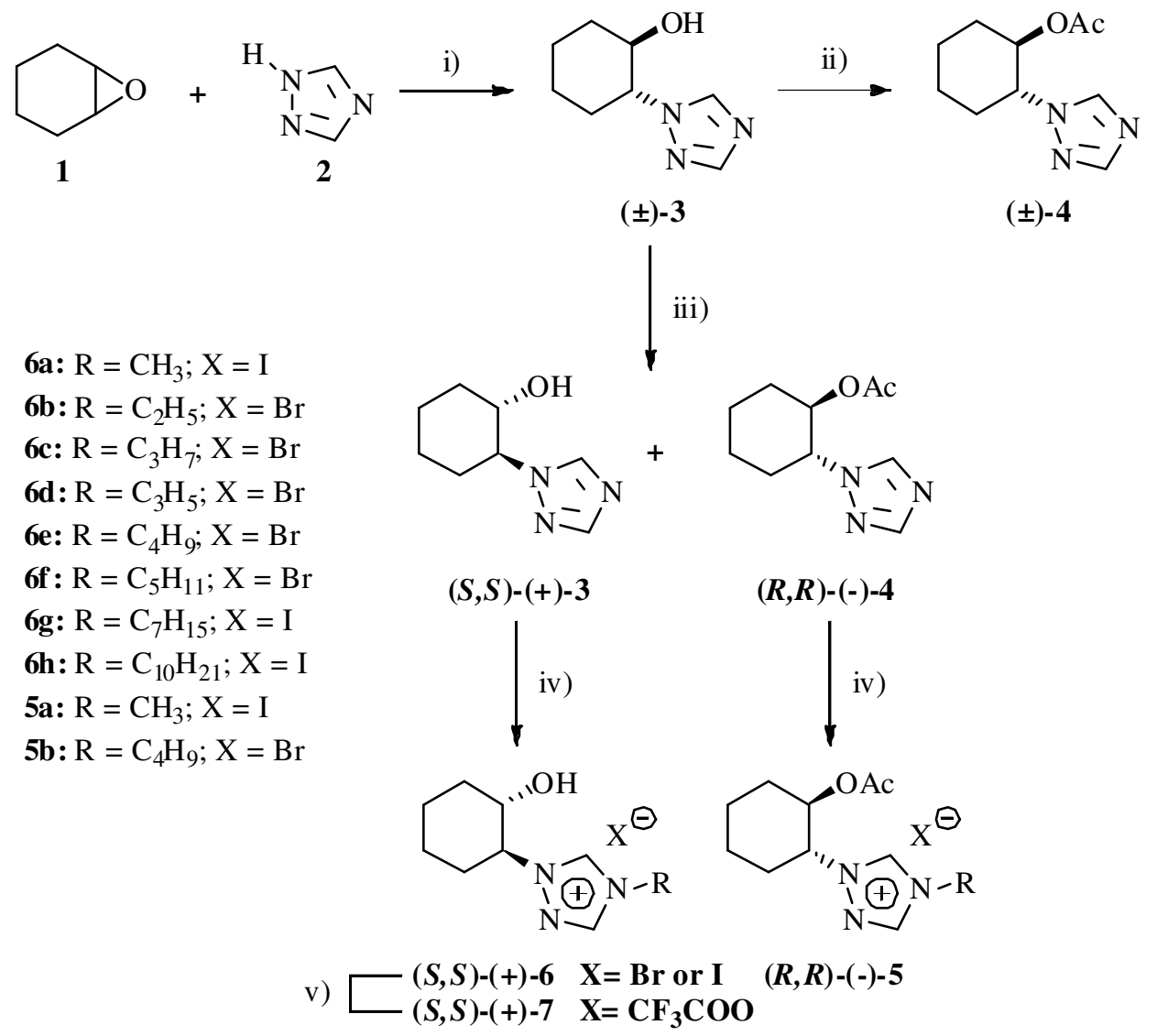

Scheme 1. Synthesis of enantiomerically enriched ILs. Reagents and conditions: (i) $65{ }^{\circ} \mathrm{C}, 72 \mathrm{~h}$; (ii) $\mathrm{Ac}_{2} \mathrm{O}$ (2 equiv), DMAP (0.33 equiv), $\mathrm{NEt}_{3}$ (3 equiv), $\mathrm{CH}_{2} \mathrm{Cl}_{2}, \mathrm{rt}, 4 \mathrm{~h}$; (iii) vinyl acetate (3 equiv), enzyme, solvent, $\mathrm{rt}$ or $30{ }^{\circ} \mathrm{C}, 250 \mathrm{rpm}$; (iv) $\mathrm{RX}, \Delta$, dry $\mathrm{CH}_{3} \mathrm{CN}$; (v) $\left(\mathrm{CF}_{3} \mathrm{COO}\right)_{2} \mathrm{~Pb}, \mathrm{H}_{2} \mathrm{O}$, rt.

Kinetic resolution of the racemic alcohol enantiomers ( \pm )-3 was performed by enantioselective acetylation catalyzed by commercially available lipases using a 3-fold molar excess of vinyl acetate as the acyl donor (Scheme 1). In preliminary studies of enzyme catalyzed acetylation of $( \pm)-3$, we have checked various solvents to establish the most convenient one for the reaction. The media employed were chloroform, MTBE, THF, 1,4-dioxane and 2-methyl-2butanol (tert-amyl alcohol). The reactions carried out in chloroform, MTBE and THF were sluggish, due to the low solubility of the substrate, and much higher reaction rates were observed in dioxane and 2-methyl-2-butanol. These two solvents were tested in order to find the proper lipase for the reaction. For that purpose, two of the most frequently used immobilized enzymes 
were examined, the lipase from: Pseudomonas cepacia (Amano PS-C) and Candida antarctica (Novozym SP 435).

In most cases, obtained enantioselectivities (Table 2) were fairly good but not fully satisfying, except for the entry rac-2. Nevertheless, the initial screening experiments reveal useful information: the ractions carried out in 2-methyl-2-butanol run faster than in dioxane, and Amano PS-C is a more effective catalyst than Novozym 435 (Table 2: entry rac-1 vs rac-3, rac-2 vs rac-4).

Table 2. Lipase-catalysed resolution of trans-( \pm )-2-(1H-1,2,4-triazol-1-yl)cyclohexanol ( \pm )-3 enantiomers

\begin{tabular}{|c|c|c|c|c|c|c|c|c|c|}
\hline Entry & Enzyme & Solvent & $\mathrm{t}, \mathrm{h}$ & $\mathrm{T},{ }^{\circ} \mathrm{C}$ & Conv., ${ }^{\mathrm{d}} \%$ & Product & $e e,{ }^{\mathrm{f}} \%$ & $E^{g}$ & Yield, ${ }^{\mathrm{h}} \%$ \\
\hline \multirow{2}{*}{$r a c-1$} & Amano & \multirow{2}{*}{ dioxane } & \multirow{2}{*}{40.5} & \multirow{2}{*}{$\mathrm{rt}$} & \multirow{2}{*}{42} & Alcohol & 61 & \multirow{2}{*}{20} & 20 \\
\hline & $P S-C^{a}$ & & & & & Ester $^{\mathrm{e}}$ & 84 & & 52 \\
\hline \multirow{2}{*}{$r a c-2$} & Amano & tert-amyl & \multirow{2}{*}{33} & \multirow{2}{*}{$\mathrm{rt}$} & \multirow{2}{*}{49} & Alcohol & 84 & \multirow{2}{*}{46} & 45 \\
\hline & PS-C ${ }^{\mathrm{a}}$ & alcohol $^{\mathrm{i}}$ & & & & Ester $^{\mathrm{e}}$ & 88 & & 28 \\
\hline \multirow{2}{*}{$r a c-3$} & Novozym & \multirow{2}{*}{ dioxane } & \multirow{2}{*}{243} & \multirow{2}{*}{$\mathrm{rt}$} & \multirow{2}{*}{50} & Alcohol & 65 & \multirow{2}{*}{9} & 60 \\
\hline & $435^{\mathrm{a}}$ & & & & & Ester $^{\mathrm{e}}$ & 65 & & 38 \\
\hline \multirow{2}{*}{$r a c-4$} & Novozym & tert-amyl & \multirow{2}{*}{47} & \multirow{2}{*}{$\mathrm{rt}$} & \multirow{2}{*}{46} & Alcohol & 74 & \multirow{2}{*}{30} & 50 \\
\hline & $435^{\mathrm{a}}$ & alcohol $^{\mathrm{i}}$ & & & & Ester $^{\mathrm{e}}$ & 87 & & 68 \\
\hline \multirow{2}{*}{$r a c-5$} & Amano & tert-amyl & \multirow{2}{*}{42} & \multirow{2}{*}{30} & \multirow{2}{*}{50} & Alcohol & 98 & \multirow{2}{*}{$>200$} & 17 \\
\hline & $P S-C^{b}$ & alcohol $^{\mathrm{i}}$ & & & & Ester $^{\mathrm{e}}$ & 98 & & 32 \\
\hline \multirow{2}{*}{$r a c-6$} & Amano & tert-amyl & \multirow{2}{*}{55} & \multirow{2}{*}{30} & \multirow{2}{*}{60} & Alcohol & 99 & \multirow{2}{*}{24} & 86 \\
\hline & PS-C $C^{\mathrm{c}}$ & alcohol $^{\mathrm{i}}$ & & & & Ester $^{\mathrm{e}}$ & 66 & & 98 \\
\hline
\end{tabular}

${ }^{\mathrm{a} C}$ Conditions: ( \pm )-3 $100 \mathrm{mg}$, lipase $30 \mathrm{mg}$, 2-methyl-2-butanol $7.5 \mathrm{ml}$, vinyl acetate $154 \mathrm{mg}$ (3 equiv).

${ }^{\mathrm{b}}$ Conditions: ( \pm )-3 $1.5 \mathrm{~g}$, lipase $0.5 \mathrm{~g}, 2$-methyl-2-butanol $75 \mathrm{ml}$, vinyl acetate $2.32 \mathrm{~g}$ (3 equiv). ${ }^{\mathrm{c} C o n d i t i o n s: ~( \pm)-3} 1 \mathrm{~g}$, lipase $0.25 \mathrm{~g}$, 2-methyl-2-butanol $50 \mathrm{ml}$, vinyl acetate $1.54 \mathrm{~g}$ (3 equiv). ${ }^{\mathrm{d}}$ based on GC [for confirmation the \% conversion was calculated from the enantiomeric excess of the unreacting alcohol $\left(e e_{\mathrm{s}}\right)$ and the product $\left(e e_{\mathrm{p}}\right)$ according to the formula conv. $=e e_{\mathrm{s}} /\left(e e_{\mathrm{s}}+\right.$ $\left.\left.e e_{\mathrm{p}}\right)\right]$.

e based on enantiomeric excess of the corresponding chiral alcohol $[(\boldsymbol{S}, \boldsymbol{S})-(+)-3]$ and conversion rate.

f based on chiral HPLC.

${ }^{\mathrm{g}} \mathrm{E}=\ln \left[(1-\mathrm{c}) \cdot\left(1-e e_{\mathrm{S}}\right)\right] / \ln \left[(1-\mathrm{c}) \cdot\left(1+e e_{\mathrm{P}}\right)\right]$.

$\mathrm{h}_{\text {isolated yield. }}$

i common name for 2-methyl-2-butanol.

Furthermore, we found that the reactions catalyzed by Amano PS-C gave a better product enantioselectivity. Thus, the most effective kinetic resolution of ( \pm )-3 was achieved in 2-methyl- 
2-butanol, at room temperature in the presence of Amano PS-C lipase. On the basis of these findings, the reaction was studied on a bigger scale and the process was slightly modified by increasing the temperature to $30{ }^{\circ} \mathrm{C}$ and terminating the experiment when $50 \%$ of conversion was achieved. Both the substrate and the product were isolated with high enantiomeric excesses $(98 \%)$ (enantioselectivity of the reaction E $>200$ ) and in satisfying yields (Table 2: entry rac-5). The best result in terms of optical purity of the residual alcohol (>99\%) was obtained when the conversion slightly exceeded $60 \%$ (Table 2: entry rac-6).

The stereochemical preference of the PS-C lipase in the acetylation reaction of ( \pm )-3 towards one of the enantiomers was determined by assignement of the absolute configuration of the unreacted alcohol $( \pm)-3$ by the method based on a double derivatization described by Mosher. ${ }^{20}$ This was achieved by transformation of the unreacted alcohol enantiomer into two diastereomeric esters by reacting it separately with the chiral auxiliaries $[(R)$ - and $(S)-\alpha$ methoxy- $\alpha$-phenylacetic acid (MPA)] and comparison of the chemical shifts in ${ }^{1} \mathrm{H}$ NMR spectra of the resulting two derivatives (Figure 1).

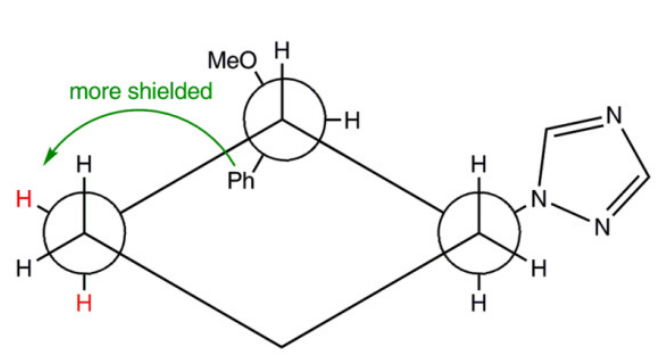

a) $(R)$-MPA ester

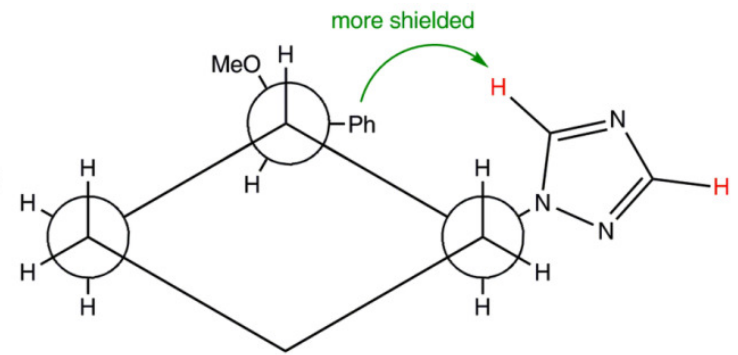

b) $(S)$-MPA ester

Figure 1. Newman projection of model for configurational correlation of MPA esters. Red marked protons are shielded by the phenyl ring of chiral auxiliary (MPA).

On the basis of the finding ${ }^{21}$ that in the $\alpha$-methoxy- $\alpha$-phenylacetic acid esters of secondary alcohols the most representative and stable conformer (by $0.6-1.0 \mathrm{kcal} / \mathrm{mol}$ ) is the sp conformer [in which the methoxy group, the $\mathrm{C}_{\alpha}$ carbon, the carbonyl group of the MPA fragment, and the $\mathrm{H}\left(7^{\prime}\right)$ hydrogen of the alcohol fragment are in the same plane (see Figure 2)], and evaluation of differences in chemical shifts of the appropriate protons in the esters, the absolute configuration of the investigated enantiomer has been assigned as $(1 S, 2 S)$.

According to Riguero et al. ${ }^{21}$ the structure can be also calculated on the basis of changes in proton chemical shifts of the asymmetric carbon substituents in the substrate $\left(\mathrm{L}_{1}\right.$ - triazole protons and $\mathrm{L}_{2}$ - protons of $-\mathrm{CH}_{2}$ group in cyclohexane ring) in both diastereisomers. These differences in the chemical shifts are represented by $\Delta \delta$ and it is the sign of this parameter (+ or -) that provides information about the configuration. For a particular substituent (e.g., $\mathrm{L}_{1}$ ), $\Delta \delta$ is defined as the difference in chemical shifts of a given signal of the substituent $\left(\delta \mathrm{L}_{1}\right)$ in the two considered spectra (diastereoisomers). 


$$
\begin{gathered}
\Delta \delta^{R S} \mathrm{~L}_{1}=\delta \mathrm{L}_{1}(R)-\delta \mathrm{L}_{1}(S)=7.98-7.68=0.30>0 \\
\Delta \delta^{R S} \mathrm{~L}_{2}=\delta \mathrm{L}_{2}(R)-\delta \mathrm{L}_{2}(S)=2.04-2.23=-0.19<0
\end{gathered}
$$

The calculated result and examination of the NMR spectra of the $(R)$ - and (S)-MPA esters are consistent with the three-dimensional structure proposed (Figure 2).
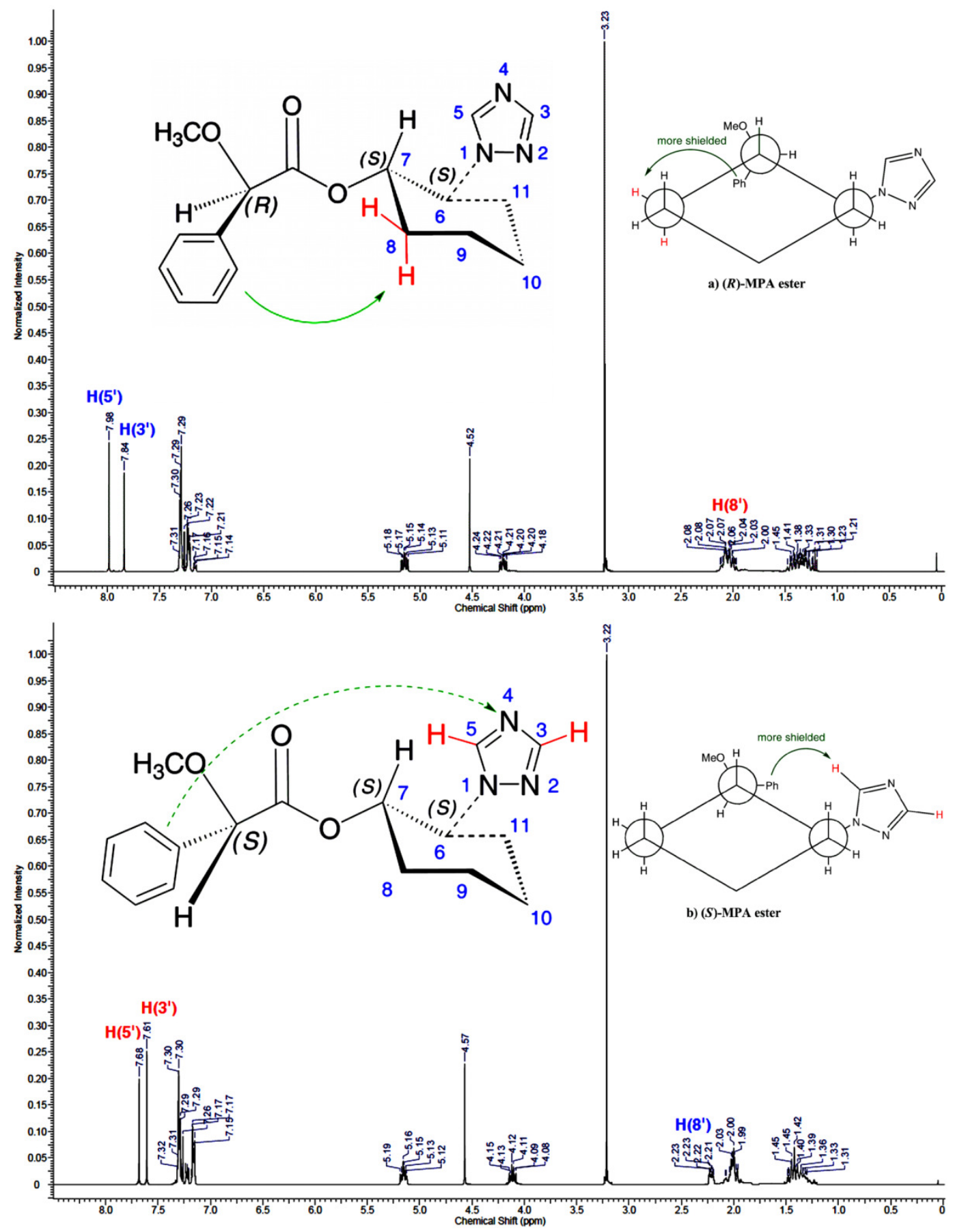

Figure 2. The assignment of absolute configuration of (+)-3 (slower reacting enantiomer) by ${ }^{1} \mathrm{H}$ NMR spectra and $\Delta \delta^{R S}$ values of $(R)$ and $(S)$-MPA esters. 
It is obvious that if the absolute configuration of the slower reacting enantiomer of $( \pm)-3$ is $(S)$ on $\mathrm{C}\left(7^{\prime}\right)$ carbon, both protons in the triazole ring $\mathrm{H}\left(3^{\prime}\right)$ and $\mathrm{H}\left(5^{\prime}\right)$ of the $(S)$-MPA ester are shielded by the phenyl ring (due to the space-orientated anisotropic effect) while the same protons in the $(R)$-MPA ester remain unaffected. The opposite effect is observed for aliphatic protons $\mathrm{H}\left(8^{\prime}\right)$ which are shielded in $(R)$-MPA derivative while in $(S)$-MPA remain unaffected. The suggested assignment is also in agreement with Kazlauskas rule, ${ }^{22}$ according to which the preferably accepted enantiomer in lipase catalyzed transesterification reactions posses $(R)$ configuration on the alcoholic center.

In Figure 3 the molecular structure of (+)-3 is shown. The crystal structure was chiral but not polar and was composed of antiparallel polar hydrogen-bonded helical chains passing in the $b$ crystallographic direction with the intermolecular hydrogen bonds $\mathrm{O}(12)-\mathrm{H}(12) \cdots \mathrm{N}(4)$ between neighboring molecules related by two-fold screw axes symmetry. The respective dimensions were $\mathrm{O}(12)-\mathrm{H}(12)=0.87(2), \mathrm{H}(12) \cdots \mathrm{N}(4)=1.97(2) \AA$ and the $\mathrm{O}-\mathrm{H} \cdots \mathrm{N}=179(2)^{\circ}$.

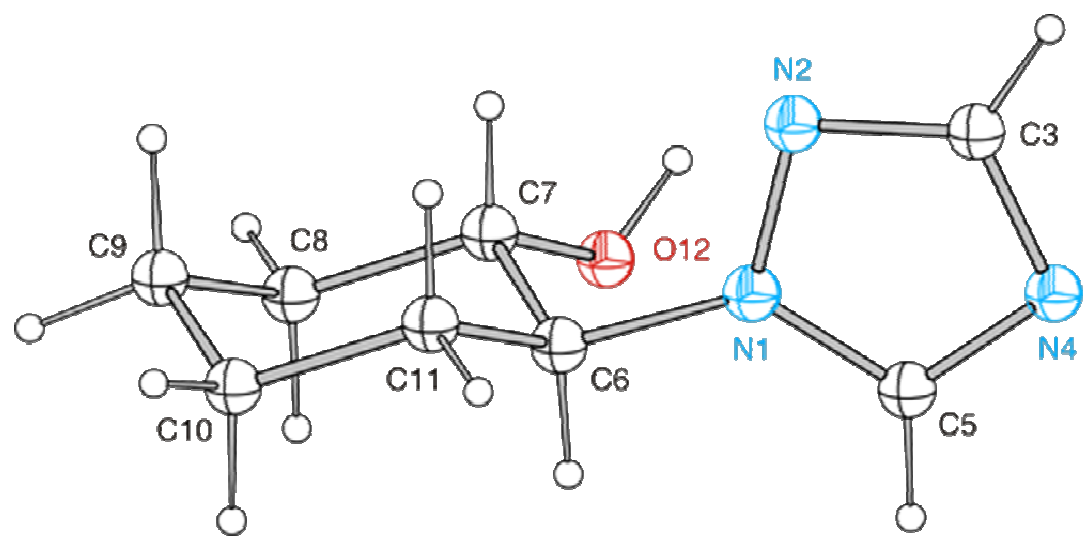

Figure 3. ORTEP view of (+)-3 molecule.

The final optically active triazolium ionic liquids were obtained by selective quaternization of the triazole rings in reactions between $(1 S, 2 S)-2-(1 H-1,2,4$-triazol-1-yl)cyclohexanol or $(1 R, 2 R)-2-(1 H$-triazol-1-yl)cyclohexyl acetate and a few different haloalkanes. As is described by several authors ${ }^{23}$ the quaternization of 1 -substituted 1,2,4-triazole ring occurres selectively at $N-4$. Two points are noteworthy at this stage. If the alkylation reaction of $(1 S, 2 S)-2-(1 H$-triazol1-yl)cyclohexanol (+)-3 was performed in neat methyl iodide used with considerable excess, $O$ methylation of the hydroxyl group was parallely observed to the $N$-methylation of the triazole ring (Table 3: entry 2). The second notice concerns the reaction of (+)-3 with ethyl bromide, which can be performed successfully only in pressurized reactor (Table 3: entry 3). 
Table 3. Synthesis and structural properties of chiral CILs

\begin{tabular}{cccccccccc}
\hline Entry & $\mathrm{IL}$ & $\mathrm{R}$ & $\mathrm{X}$ & Solvent & $\mathrm{t}, \mathrm{h}$ & $\mathrm{T},{ }^{\circ} \mathrm{C}$ & Yield, \% & $\mathrm{Mp},{ }^{\circ} \mathrm{C}$ & $\begin{array}{c}\text { Specific } \\
\text { rotation }^{\mathbf{c}}[\alpha]_{\mathrm{D}}{ }^{26}\end{array}$ \\
\hline 1 & $\mathbf{5 a}$ & $\mathrm{CH}_{3}$ & $\mathrm{I}$ & - & 48 & 42.5 & 99 & $167-168.5$ & -15.6 \\
2 & $\mathbf{6 a}^{\mathbf{a}}$ & $\mathrm{CH}_{3}$ & $\mathrm{I}$ & - & 24 & 42.5 & 62 & $191-192$ & +13.5 \\
3 & $\mathbf{6 b}^{\mathbf{b}}$ & $\mathrm{C}_{2} \mathrm{H}_{5}$ & $\mathrm{Br}$ & $\mathrm{CH}_{3} \mathrm{CN}$ & 24 & 82 & 57 & $184-185$ & +11.6 \\
4 & $\mathbf{6 c}$ & $\mathrm{C}_{3} \mathrm{H}_{7}$ & $\mathrm{Br}$ & $\mathrm{CH}_{3} \mathrm{CN}$ & 96 & 65 & 80 & $173-174$ & +9.5 \\
5 & $\mathbf{6 d}$ & $\mathrm{C}_{3} \mathrm{H}_{5}$ & $\mathrm{Br}$ & $\mathrm{CH}_{3} \mathrm{CN}$ & 72 & 63 & 80 & $164-165$ & +10.8 \\
6 & $\mathbf{6 e}$ & $\mathrm{C}_{4} \mathrm{H}_{9}$ & $\mathrm{Br}$ & $\mathrm{CH}_{3} \mathrm{CN}$ & 72 & 82 & 45 & $190-191$ & +12.2 \\
7 & $\mathbf{5 b}$ & $\mathrm{C}_{4} \mathrm{H}_{9}$ & $\mathrm{Br}$ & $\mathrm{CH}_{3} \mathrm{CN}$ & 29 & 82 & 39 & $106-108$ & -13.4 \\
8 & $\mathbf{6}$ & $\mathrm{C}_{5} \mathrm{H}_{11}$ & $\mathrm{Br}$ & $\mathrm{CH}_{3} \mathrm{CN}$ & 96 & 82 & 82 & $193-195$ & +9.7 \\
9 & $\mathbf{6 g}$ & $\mathrm{C}_{7} \mathrm{H}_{15}$ & $\mathrm{I}$ & $\mathrm{CH}_{3} \mathrm{CN}$ & 96 & 82 & 96 & $149-150$ & +7.5 \\
10 & $\mathbf{6}$ & $\mathrm{C}_{10} \mathrm{H}_{21}$ & $\mathrm{I}$ & $\mathrm{CH}_{3} \mathrm{CN}$ & 96 & 82 & 99 & $156-156.5$ & +7.8 \\
\hline
\end{tabular}

a this product is $O$-methylated iodide salt of 2-(1H-1,2,4-triazol-1-yl)cyclohexanol.

${ }^{\mathrm{b}}$ reaction was performed in pressure reactor.

${ }^{\mathrm{c}} c$ solution in chloroform $(c 1.5)$.

The synthesized triazole salts with halogen anions $(\mathbf{5 a}-\mathbf{b} ; \mathbf{6} \mathbf{6}-\mathbf{h})$ are solid. The exchange of bromide anion to the trifluoroacetate with use of $\left(\mathrm{CF}_{3} \mathrm{COO}\right)_{2} \mathrm{~Pb}$ led to the corresponding liquid at room temperature salts 7a-d, obtained with excellent isolated yields (94-99\%) (Table 4).

Table 4. Properties of CILs after anion metathesis in triazolium salts

\begin{tabular}{cccccc}
\hline Entry & $\mathrm{IL}$ & $\mathrm{R}$ & Yield, \% & $T_{\mathrm{g},}{ }^{\circ} \mathrm{C}^{\mathrm{a}}$ & $\begin{array}{c}\text { Specific rotation }^{\mathrm{b}} \\
{[\alpha]_{\mathrm{D}}{ }^{23.5}}\end{array}$ \\
\hline 1 & $\mathbf{7 a}$ & $\mathrm{C}_{2} \mathrm{H}_{5}$ & 96 & -17.5 & $+6.5(c 1.07)$ \\
2 & $\mathbf{7 b}$ & $\mathrm{C}_{3} \mathrm{H}_{7}$ & 98 & -9 & $+2.3(c 1.3)$ \\
3 & $\mathbf{7 c}$ & $\mathrm{C}_{3} \mathrm{H}_{5}$ & 94 & -10.7 & $+4.8(c 1.03)$ \\
4 & $\mathbf{7 d}$ & $\mathrm{C}_{4} \mathrm{H}_{9}$ & 99 & -13.6 & $+1.6(c 1.27)$ \\
\hline
\end{tabular}

${ }^{a}$ Glass transition temperature; the data were determined by DSC.

${ }^{\mathrm{b}} c$ solution in chloroform.

DSC measurements exhibited that these novel CILs have a glass transition temperature $\left(T_{\mathrm{g}}\right)$ ranging from -17 to $-9{ }^{\circ} \mathrm{C}$. Triazolium salts containing trifluoroacetate as a counteranion can be considered to be chiral RTILs since DSC plots are unambiguously characteristic for amorphous materials for all the cases studied. DSC determinations revealed that all CILs had good thermal stabilities up to at least $150{ }^{\circ} \mathrm{C}$. The corresponding DSC traces are shown in Figure 4. 


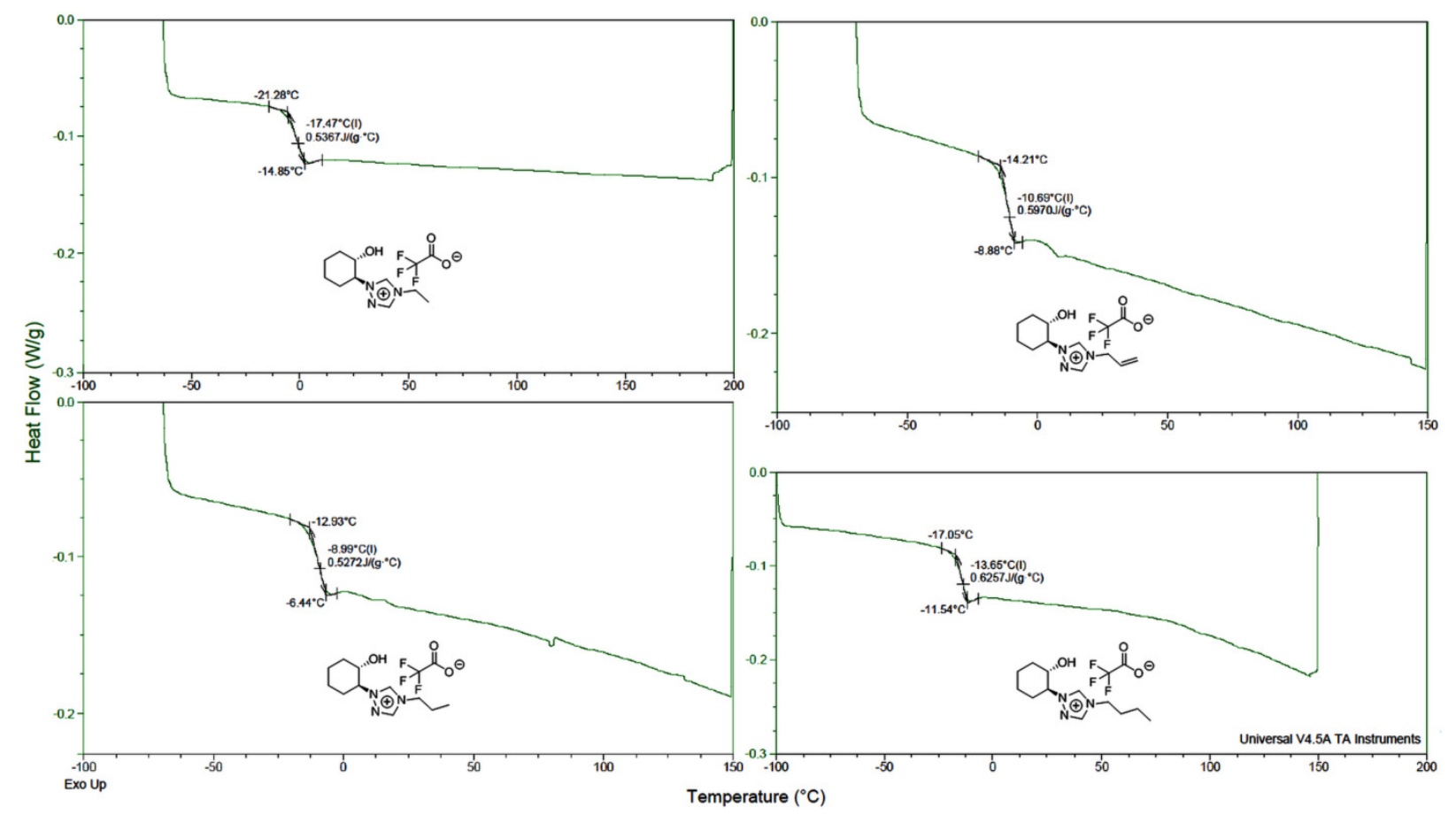

Figure 4. DSC plots for a family of enantiopure chiral triazolium salts.

\section{Conclusions}

New types of optically active, liquid at room temperature triazolium salts have been prepared. Lipase-catalyzed transesterification was established as a simple, efficient and straightforward technique for the kinetic resolution of trans- $( \pm)-2-(1 H-1,2,4-$ triazol-1-yl)cyclohexanol. Due to the method based on the double derivatization, the absolute configuration of the chiral intermediate product of CILs was deduced as (1S,2S)-2-(1H-1,2,4-triazol-1-yl)cyclohexanol. Furthermore, an X-ray diffraction analysis of a single crystal of alcohol (+)-3 unambiguously confirmed the proposed chemical structure as the $(1 S, 2 S)$-configuration. Until now, very few racemic and enantiomerically pure triazolium quaternary salts with melting points below $100{ }^{\circ} \mathrm{C}$ are known. We here report that $(1 S, 2 S)-2-(1 H-1,2,4-$ triazol-1-yl)cyclohexanol can be selectively quaternized at the $\mathrm{N}-4$ atom in the triazole ring by using a threefold molar excess of alkyl iodides or bromides yielding solid salts, which after anion exchange become liquids at room temperature. The metathesis was achieved with lead trifluoroacetate, and has proven to be a novel versatile procedure for anion exchange in ILs. Four new triazolium salts which are claimed to be ionic liquids show low glass transition temperatures and possess a high degree of thermal stability. Antibacterial and antifungal activities of the prepared compounds are being evaluated. 


\section{Experimental Section}

General. All commercially available reagents (Aldrich, Fluka and POCH) were used without further purification. Novozym SP 435 (lipase from Candida antarctica immobilized on a macroporous acrylic resin), and Amano PS-C (lipase from Pseudomonas cepacia immobilized on ceramic particles) were purchased from Novo Nordisk Co. and Amano Pharmaceutical Co. respectively and were used without any treatment. Melting points were obtained with an MPA100 Optimelt SRS apparatus. Thin-layer chromatography was carried on TLC aluminum plates with silica gel Kieselgel $60 \mathrm{~F}_{254}$ (Merck) $(0.2 \mathrm{~mm}$ thickness film) and the compounds were visualized in iodine vapors. Preparative plate chromatography was performed with DCFertigplatten Kieselgel $60 \mathrm{~F}_{254}(5 \times 20 \mathrm{~cm}$ with $0.25 \mathrm{~mm}$ thickness layer). The chromatographic analyses (GLC) were performed with an HP Series II 5890 instrument equipped with a flame ionization detector (FID) and fitted with HP-50+ $(30 \mathrm{~m})$ semipolar column. Helium $(2 \mathrm{~mL} / \mathrm{min})$ was used as carrier gas; $\mathrm{T}_{\text {injector }} 280{ }^{\circ} \mathrm{C}, \mathrm{T}_{\text {column }} 100{ }^{\circ} \mathrm{C}(3 \mathrm{~min})$ and $100-280{ }^{\circ} \mathrm{C}\left(10{ }^{\circ} \mathrm{C} / \mathrm{min}\right)$; retention times $\left(t_{R}\right)$ are given in minutes under these conditions. Column chromatography was performed using Silica gel 60 (Merck) of 40-63 $\mu \mathrm{m}$. Mixture of 95:5 $\mathrm{v} / \mathrm{v}$ chloroform/methanol was used as eluent. The enantiomeric excesses of resulting esters and alcohols were determined by HPLC analysis which were performed on a Shimadzu CTO-10ASV equipped with UV detector STD-20A and chiral column Chiralcel OD-H (Diacel), using mixtures of $n$ hexane/isopropyl alcohol as mobile phase in appropriate ratio given in experimental section; flow (f) is given in $\mathrm{mL} / \mathrm{min}$; racemic alcohols and esters were used as standards. Optical rotations were measured on a P20 polarimeter (Belligham \& Stanley Ltd., line D spectrum of sodium) in $2 \mathrm{dm}$ of length cuvette. Absorption of electromagnetic radiation waves from UV/VIS extent was made on spectrophotometer Cary 3. The X-ray data were measured using Xcalibur R Oxford Diffraction apparatus with a ccd camera-detector applying $\mathrm{CuK} \alpha$ monochromatic radiation. ${ }^{1} \mathrm{H}$ NMR and ${ }^{13} \mathrm{C}$ NMR spectra were measured on a Varian Mercury 400BB spectrometer, operating at $400 \mathrm{MHz}$ for ${ }^{1} \mathrm{H}$ nuclei and $100 \mathrm{MHz}$ for ${ }^{13} \mathrm{C}$ nuclei, if not indicated otherwise; chemical shifts $(\delta)$ are given in parts per million (ppm) related to tetramethylsilane (TMS) as internal standard; multiplicity (s, singlet; d, doublet; t, triplet; q, quartet; m, multiplet), coupling constant $(J)$ in hertz $(\mathrm{Hz})$ assignment. Mass spectra were recorded on a Micro-mass ESI Q-TOF spectrometer at the Mass Spectrometry Laboratory, Institute of Biochemistry and Biophysics (IBB), PAN. IR spectra were measured with SPECORD M80 spectrometer. Samples were prepared in paraffinic oil. Elemental analyses were performed on a Elementar Analysensysteme GmbH - VARIO EL III (Element Analyzer: CHNS). Glass transition temperatures $\left(T_{\mathrm{g}}\right)$ were recorded on a Thermal Analysis DSC Q200 differential scanning calorimeter with heating rate at $5{ }^{\circ} \mathrm{C} / \mathrm{min}$ after initially cooling samples from -70 to $150{ }^{\circ} \mathrm{C}$ under nitrogen in $\mathrm{T}_{\text {zero }}$ hermetic aluminium pans.

trans-2-(1H-1,2,4-Triazol-1-yl)cyclohexanol ( \pm )-3. 1,2,4-Triazole (7 g; $101.4 \mathrm{mmol})$ and cyclohexene oxide $(11.9 \mathrm{~g} ; 121.6 \mathrm{mmol} ; 12.3 \mathrm{ml})$ was placed in a round bottom flask and stirred 
at $65{ }^{\circ} \mathrm{C}$ for $72 \mathrm{~h}$. The resulting product was recrystallized several times from $i$-PrOH yielding white crystals (11.53 g; $68.95 \mathrm{mmol}$; Yield 68\%). Mp 128-129.5 ${ }^{\circ} \mathrm{C} ; \mathrm{R}_{f} 0.17\left[\mathrm{CHCl}_{3}-\mathrm{MeOH}\right.$ (95:5)]; ${ }^{1} \mathrm{H} \mathrm{NMR}\left(\mathrm{CDCl}_{3}, 400 \mathrm{MHz}\right) \delta: 1.35-1.44\left(\mathrm{~m}, 3 \mathrm{H}, \mathrm{CyCH}_{2}\right), 1.81-1.96\left(\mathrm{~m}, 3 \mathrm{H}, \mathrm{CyCH}_{2}\right)$, 2.06-2.17 (m, 2H, CyCH2), 3.80-3.86 (m, 1H, HOCHCHN $\left.{ }^{1}\right), 3.90-3.96\left(\mathrm{~m}, 1 \mathrm{H}, \mathrm{HOCHCHN}^{1}\right)$, $4.11(\mathrm{~s}, 1 \mathrm{H}, \mathrm{OH}), 7.81\left(\mathrm{~s}, 1 \mathrm{H}, \mathrm{CHN}^{1} \mathrm{~N}^{2}=\mathrm{CHN}^{4}\right), 8.07\left(\mathrm{~s}, 1 \mathrm{H}, \mathrm{CHN}^{1} \mathrm{CH}=\mathrm{N}^{4}\right) ;{ }^{13} \mathrm{C} \mathrm{NMR}\left(\mathrm{CDCl}_{3}\right.$, $100 \mathrm{MHz}) \quad \delta$ : $24.04\left(\mathrm{CyCH}_{2}\right), 24.67\left(\mathrm{CyCH}_{2}\right), 30.74\left(\mathrm{CyCH}_{2}\right), 33.71\left(\mathrm{CyCH}_{2}\right), 65.83$ $\left(\mathrm{HOCHCHN}{ }^{1}\right), 72.04\left(\mathrm{HOCHCHN}^{1}\right), 142.79\left(\mathrm{CHN}^{1} \mathrm{CH}=\mathrm{N}^{4}\right), 151.29\left(\mathrm{CHN}^{1} \mathrm{~N}^{2}=C \mathrm{HN}^{4}\right) ; \mathrm{MS}$ $\left(\mathrm{ESI}^{+}, \mathrm{m} / z\right)$ ): 168.129 [M $\left.\mathrm{M}^{+}, 100 \%\right]$; Anal. Calcd. (\%) for $\mathrm{C}_{8} \mathrm{H}_{13} \mathrm{~N}_{3} \mathrm{O}: \mathrm{C}, 57.46 \mathrm{H}, 7.84 \mathrm{~N}, 25.13$. Found: C, $57.48 \mathrm{H}, 7.87 \mathrm{~N}, 25.17$; IR: $v_{\mathrm{OH}} 3650-2800 \mathrm{~cm}^{-1}$; UV/VIS: $\lambda_{\max } 209 \mathrm{~nm}$; GC: $t_{R} 8.283$; HPLC [hexane- $i$-PrOH (95:5); $\mathrm{f}=0.5]: t_{R} 50.809,55.647$.

trans-2-(1H-1,2,4-Triazol-1-yl)cyclohexyl acetate ( \pm -4. Solution of $\mathrm{NEt}_{3}(0.54 \mathrm{~g} ; 5.38 \mathrm{mmol})$, acetic anhydride $(0.37 \mathrm{~g} ; 3.58 \mathrm{mmol})$ and DMAP $(72 \mathrm{mg})$ in dry $\mathrm{CH}_{2} \mathrm{Cl}_{2}(30 \mathrm{ml})$ was cooled to 0 ${ }^{\circ} \mathrm{C}$. Then 2-(1H-1,2,4-Triazol-1-yl)cyclohexanol (0.30 g; $\left.1.79 \mathrm{mmol}\right)$ was added and the reaction was stirred at room temperature during $4 \mathrm{~h}$. Next, the residue of triethylamine and solvent was evaporated under reduced pressure and the reaction was quenched with $\mathrm{H}_{2} \mathrm{O}(15 \mathrm{ml})$, and extracted with $\mathrm{CH}_{2} \mathrm{Cl}_{2}(3 \times 15 \mathrm{ml})$. The combined organic phases were dried over anhydrous $\mathrm{MgSO}_{4}$, and the solvent was evaporated under reduced pressure. The crude product was purified by column chromatography $\left[\mathrm{CHCl}_{3}-\mathrm{MeOH}\right.$ (95:5)] affording white crystals $(0.314 \mathrm{~g} ; 1.5 \mathrm{mmol}$; Yield 84\%). Mp 49-50.5 ${ }^{\circ} \mathrm{C} ; \mathrm{R}_{f} 0.55\left[\mathrm{CHCl}_{3}-\mathrm{MeOH}(95: 5)\right] ;{ }^{1} \mathrm{H} \mathrm{NMR}\left(\mathrm{CDCl}_{3}, 400 \mathrm{MHz}\right) \delta$ : 1.37-1.49 (m, 4H, $\left.\mathrm{CyCH}_{2}\right), 1.84\left(\mathrm{~s}, 3 \mathrm{H}, \mathrm{CH}_{3} \mathrm{C}=\mathrm{O}\right), 2.06-2.21\left(\mathrm{~m}, 4 \mathrm{H}, \mathrm{CyCH}_{2}\right), 4.15-4.22(\mathrm{~m}, 1 \mathrm{H}$, $\left.\operatorname{AcOCHCHN}{ }^{1}\right), 5.01-5.08\left(\mathrm{~m}, 1 \mathrm{H}, \mathrm{AcOCHCHN}^{1}\right), 7.91\left(\mathrm{~s}, 1 \mathrm{H}, \mathrm{CHN}^{1} \mathrm{~N}^{2}=\mathrm{CHN}^{4}\right), 8.10(\mathrm{~s}, 1 \mathrm{H}$, $\left.\mathrm{CHN}^{1} \mathrm{CH}=\mathrm{N}^{4}\right) ;{ }^{13} \mathrm{C} \mathrm{NMR}\left(\mathrm{CDCl}_{3}, 100 \mathrm{MHz}\right) \delta: 20.70\left(\mathrm{CyCH}_{2}\right), 23.62\left(\mathrm{CH}_{3} \mathrm{C}=\mathrm{O}\right), 24.41$ $\left(\mathrm{CyCH}_{2}\right), 31.06\left(\mathrm{CyCH}_{2}\right), 31.23\left(\mathrm{CyCH}_{2}\right), 62.28\left(\mathrm{AcOCHCHN}{ }^{1}\right), 73.71\left(\mathrm{AcOCHCHN}^{1}\right), 142.58$ $\left(\mathrm{CHN}^{1} \mathrm{CH}=\mathrm{N}^{4}\right), 151.53\left(\mathrm{CHN}^{1} \mathrm{~N}^{2}=C \mathrm{HN}^{4}\right), 169.589(C=\mathrm{O}) ; \mathrm{MS}\left(\mathrm{ESI}^{+}, \mathrm{m} / z\right)$ : $210.144 \quad\left[\mathrm{M}^{+}\right.$, 100\%]; Anal. Calcd. (\%) for $\mathrm{C}_{10} \mathrm{H}_{15} \mathrm{~N}_{3} \mathrm{O}_{2}$ : C, 57.40 H, 7.23 N, 20.08. Found: C, 57.43 H, 7.27 N, 20.11; IR: $v_{\mathrm{C}=\mathrm{O}} 1770-1710 \mathrm{~cm}^{-1}$; UV/VIS: $\lambda_{\max } 200 \mathrm{~nm}$; GC: $t_{R} 9.435$.

\section{General procedure for enzymatic resolution of racemic 2-(1H-1,2,4-triazol-1- yl)cyclohexanol ( \pm )-3}

Racemic trans-2-(1H-1,2,4-triazol-1-yl)cyclohexanol (1 g; $5.98 \mathrm{mmol})$ was dissolved in 2methyl-2-butanol $(50 \mathrm{ml})$. Then Amano PS-C lipase $(0.25 \mathrm{~g})$ and vinyl acetate $(1.54 \mathrm{~g} ; 17.94$ mmol; $1.6 \mathrm{ml}$ ) were added. The reaction mixture was shaken at $30{ }^{\circ} \mathrm{C}$ and $250 \mathrm{rpm}$. Aliquots were in 60 min. intervals analyzed by GC until $60 \%$ conversion was reached. The reaction was stopped by filtration of the enzyme. The remaining enzyme was washed with 2-methyl-2-butanol $(50 \mathrm{ml})$ and with methanol $(15 \mathrm{ml})$. The solvents were evaporated under reduced pressure and the crude product was purified by column chromatography [ $\mathrm{CHCl}_{3}-\mathrm{MeOH}$ (95:5)] affording $433 \mathrm{mg}$ of $(1 S, 2 S)-2-\left(1 H-1,2,4\right.$-triazol-1-yl)cyclohexanol [86\% isolated yield; ee $99 \% ;[\alpha]_{\mathrm{D}}{ }^{29}+39.6(\mathrm{c}=$ $\left.\left.1.0, \mathrm{CHCl}_{3}\right)\right]$ and $614 \mathrm{mg}$ of $(1 R, 2 R)-2-(1 H-1,2,4-$ triazol-1-yl)cyclohexyl acetate [98\% isolated yield; ee $\left.66 \% ;[\alpha]_{D}^{29}-28.3\left(\mathrm{c}=1.0, \mathrm{CHCl}_{3}\right)\right]$ (above procedure was given for the optimized 
conditions of enzymatic reaction; for other experiments results are presented in Table 2.; physical, spectroscopic, and analytical data are identical as for racemic standard compounds).

\section{General procedure for determination of the Absolute Configuration of (+)-3}

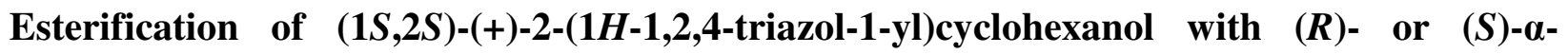
methoxy- $\alpha$-phenylacetic acid. A catalytic amount of DMAP $(5 \mathrm{mg})$ was added to a solution of $(1 S, 2 S)-(+)-2-(1 H-1,2,4-t r i a z o l-1-y l) c y c l o h e x a n o l ~(50 \mathrm{mg} ; 0.3 \mathrm{mmol})$, respectively $(R)$ - or $(S)-\alpha$ methoxy- $\alpha$-phenylacetic acid (49 mg; $0.3 \mathrm{mmol}$ ) and DCC (74 mg; $0.36 \mathrm{mmol}$ ) in dry methylene chloride $(2.5 \mathrm{ml})$. After $24 \mathrm{~h}$ of stirring at room temp., precipitated dicyclohexylurea was removed by filtration and then the urea cake was rinsed with toluene $(3 \times 0.5 \mathrm{ml})$. The combined organic solutions were washed with cold $1 \mathrm{M} \mathrm{HCl}(2 \times 1 \mathrm{ml})$, saturated $\mathrm{NaHCO}_{3}(2 \times 1 \mathrm{ml})$, and saturated $\mathrm{NaCl}(1 \times 1 \mathrm{ml})$. Then the organic layer was dried over $\mathrm{MgSO}_{4}$, filtered and the solvent was evaporated under reduced pressure. Crude product was purified by preparative plate chromatography using hexane-ethyl acetate $(1: 1)$ mixture.

(R)-[(1S,2S)-2-(1H-1,2,4-Triazol-1-yl)cyclohexyl]-2-methoxy-2-phenylacetate. Colourless oil; yield 93\%; $\mathrm{R}_{f} 0.17$ [hexane : ethyl acetate (1:1)]; ${ }^{1} \mathrm{H} \mathrm{NMR}\left(\mathrm{CDCl}_{3}, 400 \mathrm{MHz}\right) \delta: 1.21-1.45$ (m, $\left.4 \mathrm{H}, \mathrm{CyCH}_{2}\right), 1.95$ - $2.14\left(\mathrm{~m}, 4 \mathrm{H}, \mathrm{CyCH}_{2}\right), 3.23\left(\mathrm{~s}, 3 \mathrm{H}, \mathrm{OCH}_{3}\right), 4.18-4.24(\mathrm{~m}, 1 \mathrm{H}$, $\left.\mathrm{AcOCHCHN}^{1}\right), 4.52\left(\mathrm{~s}, 1 \mathrm{H}, \mathrm{PhCHOCH}_{3}\right), 5.11-5.18\left(\mathrm{~m}, 1 \mathrm{H}, \mathrm{AcOCHCHN}^{1}\right), 7.14-7.31(\mathrm{~m}, 5 \mathrm{H}$, $\mathrm{Ph}), 7.84\left(\mathrm{~s}, 1 \mathrm{H}, \mathrm{CHN}^{1} \mathrm{~N}^{2}=\mathrm{CHN}{ }^{4}\right), 7.98\left(\mathrm{~s}, 1 \mathrm{H}, \mathrm{CHN}^{1} \mathrm{CH}=\mathrm{N}^{4}\right) ;{ }^{13} \mathrm{C} \mathrm{NMR}\left(\mathrm{CDCl}_{3}, 100 \mathrm{MHz}\right) \delta$ : $23.43\left(\mathrm{CyCH}_{2}\right), 24.26\left(\mathrm{CyCH}_{2}\right), 30.56\left(\mathrm{CyCH}_{2}\right), 31.46\left(\mathrm{CyCH}_{2}\right), 57.20\left(\mathrm{OCH}_{3}\right), 61.87$ $\left(\mathrm{AcOCHCHN}{ }^{1}\right), 74.36\left(\mathrm{AcOCHCHN}^{1}\right), 82.27\left(\mathrm{PhCHOCH}_{3}\right), 126.79(3 \mathrm{C}, \mathrm{Ph}), 128.53(2 \mathrm{C}, \mathrm{Ph})$, $135.71(1 \mathrm{C}, \mathrm{Ph}), 142.54\left(\mathrm{CHN}^{1} \mathrm{~N}^{2}=C \mathrm{HN}^{4}\right), 151.66\left(\mathrm{CHN}^{1} C \mathrm{H}^{4} \mathrm{~N}^{4}\right), 169.39(C=\mathrm{O})$.

(S)-[(1S,2S)-2-(1H-1,2,4-Triazol-1-yl)cyclohexyl]-2-methoxy-2-phenylacetate. Colourless oil; yield 97\%; $\mathrm{R}_{f} 0.17$ [hexane : ethyl acetate (1:1)]; ${ }^{1} \mathrm{H} \mathrm{NMR}\left(\mathrm{CDCl}_{3}, 400 \mathrm{MHz}\right) \delta: 1.31$ - 1.45 (m, $\left.4 \mathrm{H}, \mathrm{CyCH}_{2}\right), 1.99-2.23$ (m, 4H, $\left.\mathrm{CyCH}_{2}\right), 3.22$ (s, 3H, OCH $), 4.08-4.15\left(\mathrm{~m}, 1 \mathrm{H}, \mathrm{AcOCHCHN}^{1}\right)$, $4.57\left(\mathrm{~s}, 1 \mathrm{H}, \mathrm{PhCHOCH}{ }_{3}\right), 5.12-5.19\left(\mathrm{~m}, 1 \mathrm{H}, \mathrm{AcOCHCHN}^{1}\right), 7.14-7.32(\mathrm{~m}, 5 \mathrm{H}, \mathrm{Ph}), 7.61(\mathrm{~s}$, $\left.1 \mathrm{H}, \mathrm{CHN}^{1} \mathrm{~N}^{2}=\mathrm{CHN}^{4}\right), 7.68\left(\mathrm{~s}, 1 \mathrm{H}, \mathrm{CHN}^{1} \mathrm{CH}=\mathrm{N}^{4}\right) ;{ }^{13} \mathrm{C} \mathrm{NMR}\left(\mathrm{CDCl}_{3}, 100 \mathrm{MHz}\right) \delta: 23.52$ $\left(\mathrm{CyCH}_{2}\right), 24.31\left(\mathrm{CyCH}_{2}\right), 30.98\left(\mathrm{CyCH}_{2}\right), 31.66\left(\mathrm{CyCH}_{2}\right), 57.12\left(\mathrm{OCH}_{3}\right), 61.75\left(\mathrm{AcOCHCHN}{ }^{1}\right)$, $74.31\left(\mathrm{AcOCHCHN}{ }^{1}\right), 82.31\left(\mathrm{PhCHOCH}_{3}\right), 126.77(3 \mathrm{C}, \mathrm{Ph}), 128.21(2 \mathrm{C}, \mathrm{Ph}), 135.64(1 \mathrm{C}, \mathrm{Ph})$, $142.33\left(\mathrm{CHN}^{1} \mathrm{CH}=\mathrm{N}^{4}\right), 151.50\left(\mathrm{CHN}^{1} \mathrm{~N}^{2}=C \mathrm{HN}^{4}\right), 169.43(C=\mathrm{O})$.

Crystal structure determination of (+)-3 by X-ray crystallography. Colorless, transparent crystal of dimensions $0.6681 \times 0.2881 \times 0.1741 \mathrm{~mm}$ of $(+)-3$ was used for X-ray data collection. 7465 reflections were applied to assign an orthorhombic unit cell with the dimensions: $\mathrm{a}=5.36120(10), \mathrm{b}=8.88600(10)$ and $\mathrm{c}=18.5130(2) \AA$. The unit cell has P212121 space group symmetry and the $\mathrm{Z}=4.8345$ intensities of reflections were measured (1675 independent). After solving the structure by application of SHELXS97 direct methods program (Sheldrick, G.M. SHELXS97, program for solving crystal structures, University of Goettingen, 1997, Germany), the model was consecutively refined using SHELXL97 software (Sheldrick, G.M. SHELXL97, program for crystal structur refinement, University of Goettingen, 1997, Germany). In the last 
cycles of refinement data corrected for absorption were used. The analytical absorption was used. The assigned Flack parameter of 0.0(3) [Flack H D (1983), Acta Cryst. A39, 876-881] confirmed the correctness of the absolute structure and hence the configuration of the molecule. The final R, wR and GOF were 0.0306, 0.0869 and 1.058, respectively. The detailed structural data can be found in the CSD data base under the number CCDC 830373.

4-Methyl-1-[(1R,2R)-2-acetoxycyclohexyl]-1H-1,2,4-triazol-4-ium iodide (5a). The mixture of $(1 R, 2 R)-2-(1 H-1,2,4-t r i a z o l-1-y l) c y c l o h e x y l$ acetate $(0.075 \mathrm{~g} ; 0.36 \mathrm{mmol})$ and freshly distilled methyl iodide $(0.15 \mathrm{~g} ; 1.07 \mathrm{mmol})$ was stirred during $48 \mathrm{~h}$ at $42{ }^{\circ} \mathrm{C}$. Then the reaction flask was cooled to room temperature, and the resulting yellow precipitate was filtered off, and washed with toluene $(2 \times 5 \mathrm{ml})$ affording white crystals $(0.125 \mathrm{~g}$; $0.36 \mathrm{mmol}$; Yield $99 \%)$. Mp 167-168.5 ${ }^{\circ} \mathrm{C} ; \mathrm{R}_{f} 0.11\left[\mathrm{CHCl}_{3}-\mathrm{MeOH}(95: 5)\right] ;{ }^{1} \mathrm{H} \mathrm{NMR}\left(\mathrm{CDCl}_{3}, 400 \mathrm{MHz}\right) \delta: 1.43-1.49\left(\mathrm{~m}, 2 \mathrm{H}, \mathrm{CyCH}_{2}\right)$, 1.81 - $1.94\left(\mathrm{~m}, 2 \mathrm{H}, \mathrm{CyCH}_{2}\right), 1.97\left(\mathrm{~s}, 3 \mathrm{H}, \mathrm{CH}_{3} \mathrm{C}=\mathrm{O}\right), 2.02-2.21\left(\mathrm{~m}, 2 \mathrm{H}, \mathrm{CyCH}_{2}\right), 2.33-2.37(\mathrm{~m}$, $\left.2 \mathrm{H}, \mathrm{CyCH}_{2}\right), 4.26\left(\mathrm{~s}, 3 \mathrm{H},{ }^{+} \mathrm{N}^{4} \mathrm{CH}_{3}\right), 4.68-4.74\left(\mathrm{~m}, 1 \mathrm{H}, \mathrm{AcOCHCHN}{ }^{1}\right), 5.04-5.10(\mathrm{~m}, 1 \mathrm{H}$, $\left.\mathrm{AcOCHCHN}^{1}\right), 8.94\left(\mathrm{~s}, 1 \mathrm{H}, \mathrm{CHN}^{1} \mathrm{~N}^{2}=\mathrm{CHN}^{4}\right), 11.02\left(\mathrm{~s}, 1 \mathrm{H}, \mathrm{CHN}^{1} \mathrm{CH}^{4} \mathrm{~N}^{4}\right) ;{ }^{13} \mathrm{C} \mathrm{NMR}\left(\mathrm{CDCl}_{3}\right.$, $100 \mathrm{MHz}) \delta$ : $21.40\left(\mathrm{CyCH}_{2}\right), 23.14\left(\mathrm{CH}_{3} \mathrm{C}=\mathrm{O}\right), 23.79\left(\mathrm{CyCH}_{2}\right), 30.50\left(\mathrm{CyCH}_{2}\right), 30.61\left(\mathrm{CyCH}_{2}\right)$, $35.81\left({ }^{+} \mathrm{N}^{4} \mathrm{CH}_{3}\right), 65.31\left(\mathrm{AcOCHCHN}{ }^{1}\right), 72.87\left(\mathrm{AcOCHCHN}{ }^{1}\right), 142.72\left(\mathrm{CHN}^{1} \mathrm{CH}=\mathrm{N}^{4}\right), 144.56$ $\left(\mathrm{CHN}^{1} \mathrm{~N}^{2}=C \mathrm{HN}^{4}\right), 170.06(C=\mathrm{O})$. Anal. Calcd. $(\%)$ for $\mathrm{C}_{11} \mathrm{H}_{18} \mathrm{IN}_{3} \mathrm{O}_{2}: \mathrm{C}, 37.62 \mathrm{H}, 5.17 \mathrm{~N}, 11.97$. Found: C, $37.77 \mathrm{H}, 5.02 \mathrm{~N}, 11.88 ;[\alpha]_{\mathrm{D}}{ }^{26}-15.6\left(\mathrm{c}=1.5, \mathrm{CHCl}_{3}\right)$ for ee $99 \%$.

4-Butyl-1-[(1R,2R)-2-acetoxycyclohexyl]-1H-1,2,4-triazol-4-ium bromide (5b). Optically active $(1 R, 2 R)-2-(1 H-1,2,4$-triazol-1-yl)cyclohexyl acetate $(0.1 \mathrm{~g} ; 0.48 \mathrm{mmol})$ was dissolved in dry $\mathrm{CH}_{3} \mathrm{CN}(2.5 \mathrm{ml})$ and subsequently $n$-butyl bromide $(0.065 \mathrm{~g} ; 0.48 \mathrm{mmol})$ was added. The reaction mixture was stirred in reflux for $29 \mathrm{~h}$. Reaction progress was controlled by TLC, using chloroform : methanol (95:5) mixture as the eluent. The imidazolium salt was precipitated from $\mathrm{CH}_{3} \mathrm{CN}$ by addition of toluene until cloudy at room temperature solution was obtained. The mixture was cooled to $-75{ }^{\circ} \mathrm{C}$ and stored 3 days in deep freezer. Resulting white crystals were filtered off (0.064 g; $0.12 \mathrm{mmol}$; Yield 39\%). Mp 106-108 ${ }^{\circ} \mathrm{C} ; \mathrm{R}_{f} 0.11\left[\mathrm{CHCl}_{3}-\mathrm{MeOH}(95: 5)\right]$; ${ }^{1} \mathrm{H} \mathrm{NMR}\left(\mathrm{CDCl}_{3}, 400 \mathrm{MHz}\right) \delta: 0.94\left(\mathrm{t}, J 6.8 \mathrm{~Hz}, 3 \mathrm{H}, \mathrm{CH}_{2} \mathrm{CH}_{3}\right), 1.33-1.46\left(\mathrm{~m}, 4 \mathrm{H}, \mathrm{CyCH}_{2}\right)$, $1.86-1.88\left(\mathrm{~m}, 2 \mathrm{H}, \mathrm{CyCH}_{2}\right), 1.91\left(\mathrm{~s}, 3 \mathrm{H}, \mathrm{CH}_{3} \mathrm{C}=\mathrm{O}\right), 1.94-1.98\left(\mathrm{~m}, 2 \mathrm{H}, \mathrm{CH}_{2}\right), 2.16-2.23(\mathrm{~m}$, $\left.4 \mathrm{H}, \mathrm{CH}_{2}\right), 4.59\left(\mathrm{t}, J 7.2 \mathrm{~Hz}, 2 \mathrm{H},{ }^{+} \mathrm{N}^{4} \mathrm{CH}_{2} \mathrm{CH}_{2}\right), 4.68-4.75\left(\mathrm{~m}, 1 \mathrm{H}, \mathrm{AcOCHCHN}{ }^{1}\right), 4.99-5.05$ $\left(\mathrm{m}, 1 \mathrm{H}, \mathrm{AcOCHCHN}{ }^{1}\right), 9.11\left(\mathrm{~s}, 1 \mathrm{H}, \mathrm{CHN}^{1} \mathrm{~N}^{2}=\mathrm{CHN}^{4}\right), 11.75\left(\mathrm{~s}, 1 \mathrm{H}, \mathrm{CHN}^{1} \mathrm{CH}=\mathrm{N}^{4}\right) ;{ }^{13} \mathrm{C} \mathrm{NMR}$ $\left(\mathrm{CDCl}_{3}, 100 \mathrm{MHz}\right) \delta$ : $13.31\left(\mathrm{CH}_{2} \mathrm{CH}_{3}\right), 19.18\left(\mathrm{CyCH}_{2}\right), 20.85\left(\mathrm{CH}_{2} \mathrm{CH}_{2} \mathrm{CH}_{3}\right), 23.17\left(\mathrm{CH}_{3} \mathrm{C}=\mathrm{O}\right)$, $23.78\left(\mathrm{CyCH}_{2}\right), 30.24\left(\mathrm{CH}_{2}\right), 30.57\left(\mathrm{CyCH}_{2}\right), 32.01\left(\mathrm{CyCH}_{2}\right), 48.41\left({ }^{+} \mathrm{N}^{4} \mathrm{CH}_{2} \mathrm{CH}_{2}\right), 65.25$ $\left(\mathrm{AcOCHCHN}{ }^{1}\right), 73.08\left(\mathrm{AcOCHCHN}^{1}\right), 142.99\left(\mathrm{CHN}^{1} \mathrm{~N}^{2}=\mathrm{CHN}^{4}\right), 143.82\left(\mathrm{CHN}^{1} \mathrm{CH}^{4} \mathrm{~N}^{4}\right)$, $169.83(C=\mathrm{O})$; Anal. Calcd. $(\%)$ for $\mathrm{C}_{14} \mathrm{H}_{24} \mathrm{BrN}_{3} \mathrm{O}_{2}$ : C, $48.56 \mathrm{H}, 6.99 \mathrm{~N}, 12.14$. Found: C, 48.61 $\mathrm{H}, 7.02 \mathrm{~N}, 12.11 ;[\alpha]_{\mathrm{D}}{ }^{26}-13.4\left(\mathrm{c}=1.5, \mathrm{CHCl}_{3}\right)$ for ee $99 \%$.

4-Methyl-1-[(1S,2S)-2-methoxycyclohexyl]-1H-1,2,4-triazol-4-ium iodide (6a). The mixture of $(1 S, 2 S)-2-(1 H-1,2,4$-triazol-1-yl)cyclohexanol $(0.1 \mathrm{~g} ; 0.59 \mathrm{mmol})$ and freshly distilled methyl iodide $(0.25 \mathrm{~g} ; 1.79 \mathrm{mmol})$ was stirred during $24 \mathrm{~h}$ at $42{ }^{\circ} \mathrm{C}$. Then the reaction flask was cooled at room temperature and the resulting white, fluffy precipitate was washed with $\mathrm{Et}_{2} \mathrm{O}(2 \times 5 \mathrm{ml})$ 
affording product as a white crystals (0.12 g; $0.37 \mathrm{mmol}$; Yield 62\%). Mp 191-192 ${ }^{\circ} \mathrm{C} ; \mathrm{R}_{f} 0.1$ $\left[\mathrm{CHCl}_{3}-\mathrm{MeOH}(95: 5)\right] ;{ }^{1} \mathrm{H} \mathrm{NMR}\left(\mathrm{CD}_{3} \mathrm{OD}, 400 \mathrm{MHz}\right) \delta: 1.44-1.49$ (m, 2H, $\left.\mathrm{CyCH}_{2}\right), 1.83$ - 1.89 $\left(\mathrm{m}, 2 \mathrm{H}, \mathrm{CyCH}_{2}\right), 1.97$ - $2.02\left(\mathrm{~m}, 2 \mathrm{H}, \mathrm{CyCH}_{2}\right), 2.12$ - $2.22\left(\mathrm{~m}, 2 \mathrm{H}, \mathrm{CyCH}_{2}\right), 3.77$ - $3.83(\mathrm{~m}, 1 \mathrm{H}$, $\left.\mathrm{CH}_{3} \mathrm{OCHCHN} \mathrm{N}^{1}\right), 4.02\left(\mathrm{~s}, 3 \mathrm{H}, \mathrm{OCH}_{3}\right), 4.29-4.36\left(\mathrm{~m}, 1 \mathrm{H}, \mathrm{CH}_{3} \mathrm{OCHCHN}{ }^{1}\right), 4.82(\mathrm{~s}, 3 \mathrm{H}$, $\left.{ }^{+} \mathrm{N}^{4} \mathrm{CH}_{3}\right), 8.96\left(\mathrm{~s}, 1 \mathrm{H}, \mathrm{CHN}^{1} \mathrm{~N}^{2}=\mathrm{CHN}^{4}\right), 9.98\left(\mathrm{~s}, 1 \mathrm{H}, \mathrm{CHN}{ }^{1} \mathrm{CH} H \mathrm{~N}^{4}\right) ;{ }^{13} \mathrm{C} \mathrm{NMR}\left(\mathrm{CD}_{3} \mathrm{OD}, 100\right.$ MHz) $\delta: 24.95\left(\mathrm{CyCH}_{2}\right), 25.32\left(\mathrm{CyCH}_{2}\right), 31.19\left(\mathrm{CyCH}_{2}\right), 34.97\left(\mathrm{CyCH}_{2}\right), 35.10\left({ }^{+} \mathrm{N}^{4} \mathrm{CH}_{3}\right), 48.57$ $\left(\mathrm{OCH}_{3}\right), 69.79\left(\mathrm{CH}_{3} \mathrm{OCHCHN}{ }^{1}\right), 72.11\left(\mathrm{CH}_{3} \mathrm{OCHCHN}{ }^{1}\right), 143.95\left(\mathrm{CHN}^{1} \mathrm{~N}^{2}=C \mathrm{HN}^{4}\right), 146.55$ $\left(\mathrm{CHN}^{1} \mathrm{CH}=\mathrm{N}^{4}\right)$. Anal. Calcd. $(\%)$ for $\mathrm{C}_{10} \mathrm{H}_{18} \mathrm{IN}_{3} \mathrm{O}$ : C, $37.16 \mathrm{H}, 5.61 \mathrm{~N}, 13.00$. Found: $\mathrm{C}, 37.16$ $\mathrm{H}, 5.59 \mathrm{~N}, 12.86 ;[\alpha]_{\mathrm{D}}{ }^{26}+13.5\left(\mathrm{c}=1.5, \mathrm{CHCl}_{3}\right)$ for ee $99 \%$.

4-Ethyl-1-[(1S,2S)-2-hydroxycyclohexyl]-1H-1,2,4-triazol-4-ium bromide (6b). To the solution of $(1 S, 2 S)-2-\left(1 H-1,2,4-\right.$ triazol-1-yl)cyclohexanol $(2 \mathrm{~g} ; 11.96 \mathrm{mmol})$ in dry $\mathrm{CH}_{3} \mathrm{CN}$ (6 $\mathrm{ml})$, ethyl bromide $(3.91 \mathrm{~g} ; 35.88 \mathrm{mmol} ; 2.68 \mathrm{ml})$ was added. The reaction mixture was stirred at $82{ }^{\circ} \mathrm{C}$ in acid proof stainless steel pressure reactor $\left(V=0.27 \mathrm{dm}^{3}\right)$ under $0.7 \mathrm{MPa}$ for $24 \mathrm{~h}$. Then the reaction chamber was cooled to room temperature and the excess of ethyl bromide was removed by destillation. The resulting crude product was washed with toluene $(2 \times 7.5 \mathrm{ml})$ affording gray solid (1.87 g; $6.77 \mathrm{mmol}$; Yield 57\%). Mp 184-185 ${ }^{\circ} \mathrm{C} ; \mathrm{R}_{f} 0.4\left[\mathrm{CHCl}_{3}-\mathrm{MeOH}\right.$ (8:2)]; ${ }^{1} \mathrm{H} \mathrm{NMR}\left(\mathrm{CDCl}_{3}, 400 \mathrm{MHz}\right) \delta: 1.28-1.53\left(\mathrm{~m}, 3 \mathrm{H}, \mathrm{CyCH}_{2}\right), 1.64(\mathrm{t}, J=6.95 \mathrm{~Hz}, 3 \mathrm{H}$, $\left.\mathrm{CH}_{2} \mathrm{CH}_{3}\right), 1.81\left(\mathrm{~m}, 2 \mathrm{H}, \mathrm{CyCH}_{2}\right), 1.91-2.05\left(\mathrm{~m}, 1 \mathrm{H}, \mathrm{CyCH}_{2}\right), 2.13\left(\mathrm{~m}, 2 \mathrm{H}, \mathrm{CyCH}_{2}\right), 2.68(\mathrm{~s}, 1 \mathrm{H}$, $\mathrm{OH}), 3.81\left(\mathrm{~m}, 1 \mathrm{H}, \mathrm{HOCHCHN}{ }^{1}\right), 4.52\left(\mathrm{~m}, 2 \mathrm{H},{ }^{+} \mathrm{N}^{4} \mathrm{CH}_{2} \mathrm{CH}_{3}\right), 4.84\left(\mathrm{~m}, 1 \mathrm{H}, \mathrm{HOCHCHN}^{1}\right), 8.89$

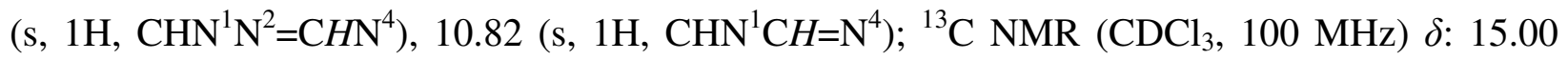

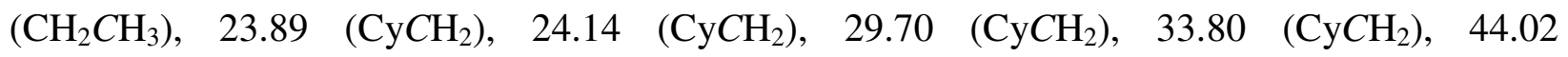
$\left({ }^{+} \mathrm{N}^{4} \mathrm{CH}_{2} \mathrm{CH}_{3}\right), 68.58\left(\mathrm{HOCHCHN}^{1}\right), 70.89\left(\mathrm{HOCHCHN}^{1}\right), 142.29\left(\mathrm{CHN}^{1} \mathrm{~N}^{2}=\mathrm{CHN}^{4}\right), 143.31$ $\left(\mathrm{CHN}^{1} \mathrm{CH}=\mathrm{N}^{4}\right)$. Anal. Calcd. (\%) for $\mathrm{C}_{10} \mathrm{H}_{18} \mathrm{BrN}_{3} \mathrm{O}$ : C, $43.49 \mathrm{H}, 6.57 \mathrm{~N}, 15.21$. Found: C, 43.44 $\mathrm{H}, 6.55 \mathrm{~N}, 15.18 ;[\alpha]_{\mathrm{D}}{ }^{26}+11.6\left(\mathrm{c}=1.5, \mathrm{CHCl}_{3}\right)$ for ee $99 \%$.

4-Propyl-1-[(1S,2S)-2-hydroxycyclohexyl]-1H-1,2,4-triazol-4-ium bromide (6c). To the solution of $(1 S, 2 S)-2-\left(1 H-1,2,4-\right.$ triazol-1-yl)cyclohexanol $(1.5 \mathrm{~g} ; 8.97 \mathrm{mmol})$ in dry $\mathrm{CH}_{3} \mathrm{CN}$ (2.5 $\mathrm{ml})$, propyl bromide $(3.3 \mathrm{~g} ; 26.9 \mathrm{mmol} ; 2.44 \mathrm{ml})$ was added. Then the reaction mixture was stirred during $96 \mathrm{~h}$ at $65^{\circ} \mathrm{C}$. Next the flask was cooled to room temperature and the excess of propyl bromide was evaporated under reduced pressure. The resulting white solid was filtered off and washed with toluene $(2 \times 5 \mathrm{ml})$ to yield colorless crystals $(2.08 \mathrm{~g} ; 7.16 \mathrm{mmol}$; Yield 80\%). mp 173-174 ${ }^{\circ} \mathrm{C} ; \mathrm{R}_{f} 0.16\left[\mathrm{CHCl}_{3}-\mathrm{MeOH}(95: 5)\right] ;{ }^{1} \mathrm{H} \mathrm{NMR}\left(\mathrm{CDCl}_{3}, 400 \mathrm{MHz}\right) \delta: 1.00(\mathrm{t}, J=7.33$ $\left.\mathrm{Hz}, 3 \mathrm{H}, \mathrm{CH}_{2} \mathrm{CH}_{3}\right), 1.29-1.54\left(\mathrm{~m}, 2 \mathrm{H}, \mathrm{CH}_{2} \mathrm{CH}_{2} \mathrm{CH}_{3}\right), 1.81\left(\mathrm{~m}, 2 \mathrm{H}, \mathrm{CyCH}_{2}\right), 1.92$ - $2.06(\mathrm{~m}, 3 \mathrm{H}$, $\left.\mathrm{CyCH}_{2}\right), 2.13\left(\mathrm{~m}, 2 \mathrm{H}, \mathrm{CyCH}_{2}\right), 2.58(\mathrm{~s}, 1 \mathrm{H}, \mathrm{OH}), 3.74-3.85\left(\mathrm{~m}, 1 \mathrm{H}, \mathrm{CyCH}_{2}\right), 4.33$ - $4.51(\mathrm{~m}$, $\left.2 \mathrm{H},{ }^{+} \mathrm{N}^{4} \mathrm{CH}_{2} \mathrm{CH}_{2} \mathrm{CH}_{3}\right), 4.52$ - $4.61\left(\mathrm{~m}, 1 \mathrm{H}, \mathrm{HOCHCHN}{ }^{1}\right), 4.75-4.86\left(\mathrm{~m}, 1 \mathrm{H}, \mathrm{HOCHCHN}^{1}\right), 8.81$

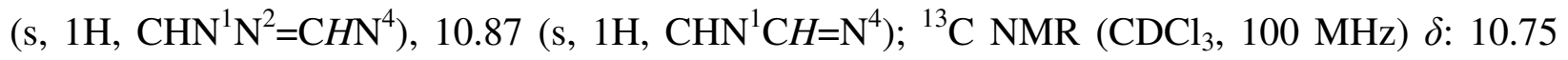
$\left(\mathrm{CH}_{2} \mathrm{CH}_{3}\right), 23.11\left(\mathrm{CH}_{2} \mathrm{CH}_{3}\right), 23.92\left(\mathrm{CyCH}_{2}\right), 24.14\left(\mathrm{CyCH}_{2}\right), 29.61\left(\mathrm{CyCH}_{2}\right), 33.81\left(\mathrm{CyCH}_{2}\right)$, $49.95\left({ }^{+} \mathrm{N}^{4} \mathrm{CH}_{2} \mathrm{CH}_{2} \mathrm{CH}_{3}\right), 68.52\left(\mathrm{HOCHCHN}^{1}\right), 70.95\left(\mathrm{HOCHCHN}^{1}\right), 142.52\left(\mathrm{CHN}^{1} \mathrm{~N}^{2}=\mathrm{CHN}^{4}\right)$, 143.45 $\left(\mathrm{CHN}^{1} \mathrm{CH}=\mathrm{N}^{4}\right)$; Anal. Calcd. $(\%)$ for $\mathrm{C}_{11} \mathrm{H}_{20} \mathrm{BrN}_{3} \mathrm{O}: \mathrm{C}, 45.53 \mathrm{H}, 6.95 \mathrm{~N}, 14.48$. Found: $\mathrm{C}$, $45.65 \mathrm{H}, 6.94 \mathrm{~N}, 14.44 ;[\alpha]_{\mathrm{D}}{ }^{26}+9.5\left(\mathrm{c}=1.5, \mathrm{CHCl}_{3}\right)$ for ee $99 \%$. 
4-(Prop-2-en-1-yl)-1-[(1S,2S)-2-hydroxycyclohexyl]-1H-1,2,4-triazol-4-ium bromide (6d). To the solution of $(1 S, 2 S)-2-(1 H-1,2,4$-triazol-1-yl)cyclohexanol $(0.7 \mathrm{~g} ; 41.86 \mathrm{mmol})$ in dry $\mathrm{CH}_{3} \mathrm{CN}(3 \mathrm{ml})$, allyl bromide $(1.52 \mathrm{~g} ; 12.56 \mathrm{mmol} ; 1.1 \mathrm{ml})$ was added. Then the reaction mixture was stirred during $72 \mathrm{~h}$ at $65^{\circ} \mathrm{C}$. Next the reaction flask was cooled to room temperature and the excess of allyl bromide was distiled off. The resulting yellowish solid was filtered off, and washed with diethyl ether $(5 \mathrm{ml})$ and toluene $(5 \mathrm{ml})$. The solid was purified by recrystallization from $\mathrm{CH}_{3} \mathrm{CN}$ to yield white crystals $(0.96 \mathrm{~g} ; 3.33 \mathrm{mmol}$; Yield $80 \%)$. Mp 164-165 ${ }^{\circ} \mathrm{C} ; \mathrm{R}_{f} 0.18$ $\left[\mathrm{CHCl}_{3}-\mathrm{MeOH}(95: 5)\right] ;{ }^{1} \mathrm{H} \mathrm{NMR}\left(\mathrm{CDCl}_{3}, 400 \mathrm{MHz}\right) \delta: 1.28-1.56(\mathrm{~m}, 4 \mathrm{H}, \mathrm{CyCH}), 1.70-2.18$ $\left(\mathrm{m}, 4 \mathrm{H}, \mathrm{CyCH}_{2}\right), 2.63(\mathrm{~s}, 1 \mathrm{H}, \mathrm{OH}), 3.72-3.86\left(\mathrm{~m}, 1 \mathrm{H}, \mathrm{HOCHCHN}^{1}\right), 4.47-4.61(\mathrm{~m}, 1 \mathrm{H}$, $\left.\mathrm{HOCHCHN}^{1}\right), 5.06-5.20\left(\mathrm{~m}, 2 \mathrm{H},{ }^{+} \mathrm{NCH}_{2} \mathrm{CH}_{\mathrm{a}}\right), 5.44-5.62\left(\mathrm{~m}, 2 \mathrm{H},{ }^{+} \mathrm{NCH}_{2} \mathrm{CH}_{\mathrm{a}}=\mathrm{CH}_{\mathrm{a}} H_{\mathrm{b}}\right), 6.10-$ $6.17\left(\mathrm{~m}, 1 \mathrm{H},{ }^{+} \mathrm{NCH}_{2} \mathrm{CH}_{\mathrm{a}}=\mathrm{CH}_{\mathrm{a}} \mathrm{H}_{\mathrm{b}}\right), 8.65\left(\mathrm{~s}, 1 \mathrm{H}, \mathrm{CHN}^{1} \mathrm{~N}^{2}=\mathrm{CHN} \mathrm{N}^{4}\right), 10.80\left(\mathrm{~s}, 1 \mathrm{H}, \mathrm{CHN}^{1} \mathrm{CH}^{4} \mathrm{~N}^{4}\right)$; ${ }^{13} \mathrm{C} \mathrm{NMR}\left(\mathrm{CDCl}_{3}, 100 \mathrm{MHz}\right) \delta$ : $23.88\left(\mathrm{CyCH}_{2}\right), 24.12\left(\mathrm{CyCH}_{2}\right), 29.49\left(\mathrm{CyCH}_{2}\right), 33.68\left(\mathrm{CyCH}_{2}\right)$, $50.72\left({ }^{+} \mathrm{NCH}_{2} \mathrm{CH}_{\mathrm{a}}\right), 68.66\left(\mathrm{HOCHCHN}^{1}\right), 70.98\left(\mathrm{HOCHCHN}^{1}\right), 123.73\left({ }^{+} \mathrm{NCH}_{2} \mathrm{CH}_{\mathrm{a}}=\mathrm{CH}_{\mathrm{a}} \mathrm{H}_{\mathrm{b}}\right)$, $128.96\left({ }^{+} \mathrm{NCH}_{2} \mathrm{CH}_{\mathrm{a}}=\mathrm{CH}_{\mathrm{a}} \mathrm{H}_{\mathrm{b}}\right), 142.47\left(\mathrm{CHN}^{1} \mathrm{~N}^{2}=C \mathrm{HN}^{4}\right), 143.12\left(\mathrm{CHN}^{1} \mathrm{CH}^{4} \mathrm{~N}^{4}\right)$; Anal. Calcd. (\%) for $\mathrm{C}_{11} \mathrm{H}_{18} \mathrm{BrN}_{3} \mathrm{O}$ : C, $45.84 \mathrm{H}, 6.30 \mathrm{~N}, 14.58$. Found: C, $45.79 \mathrm{H}, 6.28 \mathrm{~N}, 14.52$; $[\alpha]_{\mathrm{D}}{ }^{26}+$ $10.8\left(\mathrm{c}=1.5, \mathrm{CHCl}_{3}\right)$ for ee $99 \%$.

4-Butyl-1-[(1S,2S)-2-hydroxycyclohexyl]-1H-1,2,4-triazol-4-ium bromide (6e). To the solution of $(1 S, 2 S)-2-\left(1 H-1,2,4\right.$-triazol-1-yl)cyclohexanol $(0.7 \mathrm{~g} ; 41.86 \mathrm{mmol})$ in dry $\mathrm{CH}_{3} \mathrm{CN}$ ( $2.5 \mathrm{ml})$, butyl bromide $(1.72 \mathrm{~g} ; 12.56 \mathrm{mmol} ; 1.35 \mathrm{ml})$ was added. Then the reaction mixture was stirred during $72 \mathrm{~h}$ at $80^{\circ} \mathrm{C}$. After cooling to room temperature the excess of butyl bromide was distiled off. The resulting yellowish solid was filtered off, and washed with diethyl ether (3 ml) and toluene $(3 \mathrm{ml})$. The product was purified by recrystallization from $\mathrm{CH}_{3} \mathrm{CN}$ to yield white crystals (0.574 g; $1.88 \mathrm{mmol}$; Yield 45\%). Mp 190-191 ${ }^{\circ} \mathrm{C} ; \mathrm{R}_{f} 0.19\left[\mathrm{CHCl}_{3}-\mathrm{MeOH}(95: 5)\right] ;{ }^{1} \mathrm{H}$ NMR $\left(\mathrm{CDCl}_{3}, 400 \mathrm{MHz}\right) \delta: 0.95\left(\mathrm{t}, J=7.45 \mathrm{~Hz}, 3 \mathrm{H}, \mathrm{CH}_{2} \mathrm{CH}_{3}\right), 1.28-1.57\left(\mathrm{~m}, 5 \mathrm{H}, \mathrm{CH}_{2}\right), 1.81-$ $1.85\left(\mathrm{~m}, 2 \mathrm{H}, \mathrm{CH}_{2}\right), 1.90-2.04\left(\mathrm{~m}, 3 \mathrm{H}, \mathrm{CH}_{2}\right), 2.07-2.20\left(\mathrm{~m}, 2 \mathrm{H}, \mathrm{CH}_{2}\right), 3.74-3.85(\mathrm{~m}, 1 \mathrm{H}$, $\left.\mathrm{HOCHCHN}^{1}\right), 4.36-4.64\left(\mathrm{~m}, 2 \mathrm{H},{ }^{+} \mathrm{N}^{4} \mathrm{CH}_{2}\right), 4.87-4.91\left(\mathrm{~m}, 1 \mathrm{H}, \mathrm{HOCHCHN}^{1}\right), 8.74(\mathrm{~s}, 1 \mathrm{H}$,

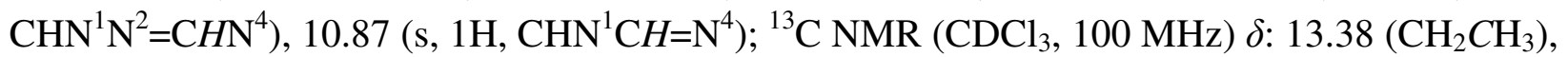
$19.51\left(\mathrm{CH}_{2} \mathrm{CH}_{3}\right), 23.93\left(\mathrm{CyCH}_{2}\right), 24.12\left(\mathrm{CyCH}_{2}\right), 29.59\left(\mathrm{CH}_{2} \mathrm{CH}_{2} \mathrm{CH}_{3}\right), 31.47\left(\mathrm{CyCH}_{2}\right), 33.78$ $\left(\mathrm{CyCH}_{2}\right), 48.37\left({ }^{+} \mathrm{N}^{4} \mathrm{CH}_{2} \mathrm{CH}_{2} \mathrm{CH}_{2} \mathrm{CH}_{3}\right), 68.50\left(\mathrm{HOCHCHN}{ }^{1}\right), 70.98\left(\mathrm{HOCHCHN}^{1}\right), 142.56$ $\left(\mathrm{CHN}^{1} \mathrm{~N}^{2}=C \mathrm{HN}^{4}\right), 143.33\left(\mathrm{CHN}^{1} C \mathrm{H}^{4} \mathrm{~N}^{4}\right)$; Anal. Calcd. $(\%)$ for $\mathrm{C}_{12} \mathrm{H}_{22} \mathrm{BrN}_{3} \mathrm{O}: \mathrm{C}, 47.38 \mathrm{H}, 7.29$ $\mathrm{N}, 13.81$. Found: $\mathrm{C}, 47.26 \mathrm{H}, 7.21 \mathrm{~N}, 13.73 ;[\alpha]_{\mathrm{D}}{ }^{26}+12.2\left(\mathrm{c}=1.5, \mathrm{CHCl}_{3}\right)$ for ee $99 \%$.

4-Pentyl-1-[(1S,2S)-2-hydroxycyclohexyl]-1H-1,2,4-triazol-4-ium bromide (6f). To the solution of $(1 S, 2 S)-2-\left(1 H-1,2,4-\right.$ triazol-1-yl)cyclohexanol $(0.5 \mathrm{~g} ; 2.99 \mathrm{mmol})$ in dry $\mathrm{CH}_{3} \mathrm{CN}$ $(6 \mathrm{ml}), n$-amyl bromide $(1.35 \mathrm{~g} ; 8.97 \mathrm{mmol} ; 1.11 \mathrm{ml})$ was added. Then the reaction mixture was stirred during $96 \mathrm{~h}$ at $82{ }^{\circ} \mathrm{C}$, and cooled to room temperature. The resulting gray solid was filtered off, and washed with diethyl ether $(2 \times 2 \mathrm{ml})$, yielding gray solid product $(0.78 \mathrm{~g} ; 2.46$ mmol; 82\%). Mp 193-195 ${ }^{\circ} \mathrm{C} ; \mathrm{R}_{f} 0.14\left[\mathrm{CHCl}_{3}-\mathrm{MeOH}(95: 5)\right] ;{ }^{1} \mathrm{H} \mathrm{NMR}\left(\mathrm{CDCl}_{3}, 400 \mathrm{MHz}\right) \delta$ : $0.90\left(\mathrm{t}, J=8 \mathrm{~Hz}, 3 \mathrm{H}, \mathrm{CH}_{2} \mathrm{CH}_{3}\right), 1.27-1.59\left(\mathrm{~m}, 8 \mathrm{H}, \mathrm{CH}_{2}\right), 1.74-2.07\left(\mathrm{~m}, 6 \mathrm{H}, \mathrm{CH}_{2}\right), 2.15(\mathrm{~m}$, $\left.2 \mathrm{H}, \mathrm{CH}_{2}\right), 3.73-3.87\left(\mathrm{~m}, 1 \mathrm{H}, \mathrm{HOCHCH \textrm {N } ^ { 1 }}\right), 4.34-4.56\left(\mathrm{~m}, 2 \mathrm{H},{ }^{+} \mathrm{N}^{4} \mathrm{CH}_{2}\right), 4.57-4.67(\mathrm{~m}, 1 \mathrm{H}$, $\left.\mathrm{HOCHCHN}^{1}\right), 8.61\left(\mathrm{~s}, 1 \mathrm{H}, \mathrm{CHN}^{1} \mathrm{~N}^{2}=\mathrm{CHN}^{4}\right), 10.94\left(\mathrm{~s}, 1 \mathrm{H}, \mathrm{CHN}^{1} \mathrm{CH}^{4} \mathrm{~N}^{4}\right) ;{ }^{13} \mathrm{C} \mathrm{NMR}\left(\mathrm{CDCl}_{3}\right.$, 
$100 \mathrm{MHz}) \delta$ : $13.79\left(\mathrm{CH}_{2} \mathrm{CH}_{3}\right), 21.99\left(\mathrm{CyCH}_{2}\right), 23.98\left(\mathrm{CH}_{2} \mathrm{CH}_{3}\right), 24.17\left(\mathrm{CH}_{2} \mathrm{CH}_{2} \mathrm{CH}_{2} \mathrm{CH}_{3}\right), 28.28$ $\left(\mathrm{CyCH}_{2}\right), 29.34\left(\mathrm{CH}_{2} \mathrm{CH}_{2} \mathrm{CH}_{2} \mathrm{CH}_{3}\right), 29.63\left(\mathrm{CyCH}_{2}\right), 33.81\left(\mathrm{CyCH}_{2}\right), 48.70\left({ }^{+} \mathrm{N}^{4} \mathrm{CH}_{2}\right), 68.59$ $\left(\mathrm{HOCHCHN}{ }^{1}\right), 71.06\left(\mathrm{HOCHCHN}^{1}\right), 142.71\left(\mathrm{CHN}^{1} \mathrm{~N}^{2}=C \mathrm{HN}^{4}\right), 143.11\left(\mathrm{CHN}^{1} \mathrm{CH}=\mathrm{N}^{4}\right)$; Anal. Calcd. (\%) for $\mathrm{C}_{13} \mathrm{H}_{24} \mathrm{BrN}_{3} \mathrm{O}: \mathrm{C}, 49.06 \mathrm{H}, 7.60 \mathrm{~N}, 13.20$. Found: C, $48.96 \mathrm{H}, 7.69 \mathrm{~N}, 13.21$; $[\alpha]_{\mathrm{D}}^{26}+9.7\left(\mathrm{c}=1.5, \mathrm{CHCl}_{3}\right)$ for ee $99 \%$.

4-Heptyl-1-[(1S,2S)-2-hydroxycyclohexyl]-1H-1,2,4-triazol-4-ium iodide (6g). To the solution of $(1 S, 2 S)-2-\left(1 H-1,2,4\right.$-triazol-1-yl)cyclohexanol $(0.5 \mathrm{~g} ; 2.99 \mathrm{mmol})$ in dry $\mathrm{CH}_{3} \mathrm{CN}(6 \mathrm{ml})$, heptyl iodide ( $2 \mathrm{~g} ; 8.97 \mathrm{mmol} ; 1.47 \mathrm{ml}$ ) was added. Then the reaction mixture was stirred during $96 \mathrm{~h}$ at $82{ }^{\circ} \mathrm{C}$. Next the reaction flask was put into icebox and cooled until yellow solid has crystallized. The resulting yellowish solid was filtered off and washed with diethyl ether ( $3 \mathrm{ml})$. Next yellowish solid was dissolved in warm $i-\mathrm{PrOH}$ and precipitated by diethyl ether to give white crystals (1.12 g; $2.86 \mathrm{mmol} ; 96 \%)$. Mp 149-150 ${ }^{\circ} \mathrm{C} ; \mathrm{R}_{f} 0.13\left[\mathrm{CHCl}_{3}-\mathrm{MeOH}(95: 5)\right] ;{ }^{1} \mathrm{H}$ NMR $\left(\mathrm{CDCl}_{3}, 400 \mathrm{MHz}\right) \delta: 0.86\left(\mathrm{t}, J=6.78 \mathrm{~Hz}, 3 \mathrm{H}, \mathrm{CH}_{2} \mathrm{CH}_{3}\right), 1.20-1.45\left(\mathrm{~m}, 10 \mathrm{H}, \mathrm{CH}_{2}\right), 1.81-$ $1.87\left(\mathrm{~m}, 2 \mathrm{H}, \mathrm{CH}_{2}\right), 1.91-2.08\left(\mathrm{~m}, 4 \mathrm{H}, \mathrm{CH}_{2}\right), 2.10-2.24\left(\mathrm{~m}, 2 \mathrm{H}, \mathrm{CH}_{2}\right), 3.80-3.93(\mathrm{~m}, 1 \mathrm{H}$, $\left.\mathrm{HOCHCHN}^{1}\right), 4.34-4.56\left(\mathrm{~m}, 2 \mathrm{H},{ }^{+} \mathrm{N}^{4} \mathrm{CH}_{2}\right), 4.62$ - $4.74\left(\mathrm{~m}, 1 \mathrm{H}, \mathrm{HOCHCHN}^{1}\right), 8.59(\mathrm{~s}, 1 \mathrm{H}$,

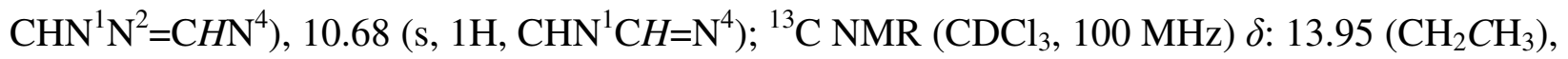
$22.42 \quad\left(\mathrm{CyCH}_{2}\right), \quad 23.93 \quad\left(\mathrm{CH}_{2} \mathrm{CH}_{3}\right), \quad 24.07 \quad\left(\mathrm{CyCH}_{2}\right), \quad 26.20 \quad\left({ }^{+} \mathrm{N}^{4} \mathrm{CH}_{2} \mathrm{CH}_{2} \mathrm{CH}_{2}\right), \quad 28.53$ $\left(\mathrm{CH}_{2} \mathrm{CH}_{2} \mathrm{CH}_{2} \mathrm{CH}_{3}\right), 29.51\left(\mathrm{CyCH}_{2}\right), 29.58\left({ }^{+} \mathrm{N}^{4} \mathrm{CH}_{2} \mathrm{CH}_{2}\right), 31.45\left(\mathrm{CH}_{2} \mathrm{CH}_{2} \mathrm{CH}_{3}\right), 33.77\left(\mathrm{CyCH}_{2}\right)$, $48.98\left({ }^{+} \mathrm{N}^{4} \mathrm{CH}_{2}\right), 68.50\left(\mathrm{HOCHCHN}^{1}\right), 70.92\left(\mathrm{HOCHCHN}^{1}\right), 142.22\left(\mathrm{CHN}^{1} \mathrm{~N}^{2}=\mathrm{CHN}^{4}\right), 143.18$ $\left(\mathrm{CHN}^{1} \mathrm{CH}=\mathrm{N}^{4}\right)$; Anal. Calcd. $(\%)$ for $\mathrm{C}_{15} \mathrm{H}_{28} \mathrm{IN}_{3} \mathrm{O}: \mathrm{C}, 45.81 \mathrm{H}, 7.18 \mathrm{~N}, 10.68$. Found: $\mathrm{C}, 45.65$ $\mathrm{H}, 7.21 \mathrm{~N}, 10.74 ;[\alpha]_{\mathrm{D}}{ }^{26}+7.5\left(\mathrm{c}=1.5, \mathrm{CHCl}_{3}\right)$ for ee $99 \%$.

4-Decyl-1-[(1S,2S)-2-hydroxycyclohexyl]-1H-1,2,4-triazol-4-ium iodide $(6 \mathrm{~h})$. To the solution of (1S,2S)-2-(1H-1,2,4-triazol-1-yl)cyclohexanol (0.5 g; $2.99 \mathrm{mmol})$ in dry $\mathrm{CH}_{3} \mathrm{CN}(6 \mathrm{ml})$, decyl iodide $(2.4 \mathrm{~g} ; 8.97 \mathrm{mmol} ; 1.91 \mathrm{ml})$ was added. Then the reaction mixture was stirred during $96 \mathrm{~h}$ at $82{ }^{\circ} \mathrm{C}$. Next the reaction flask was immersed in an icebox and cooled until yellow solid has crystallized. The resulting yellowish precipitate was filtered off and washed with diethyl ether (3 $\mathrm{ml})$. Next, the precipitate was dissolved in warm $i$-PrOH and repeatedly precipitated by diethyl ether addition to give white crystals $(1.29 \mathrm{~g} ; 2.97 \mathrm{mmol} ; 99 \%)$. Mp 156-156.5 ${ }^{\circ} \mathrm{C} ; \mathrm{R}_{f} 0.1\left[\mathrm{CHCl}_{3^{-}}\right.$ $\mathrm{MeOH}(95: 5)] ;{ }^{1} \mathrm{H} \mathrm{NMR}\left(\mathrm{CDCl}_{3}, 400 \mathrm{MHz}\right) \delta: 0.86\left(\mathrm{t}, J=6.89 \mathrm{~Hz}, 3 \mathrm{H}, \mathrm{CH}_{2} \mathrm{CH}_{3}\right), 1.16-1.49$ $\left(\mathrm{m}, 16 \mathrm{H}, \mathrm{CH}_{2}\right), 1.77$ - $1.92\left(\mathrm{~m}, 3 \mathrm{H}, \mathrm{CH}_{2}\right), 1.93-2.08\left(\mathrm{~m}, 3 \mathrm{H}, \mathrm{CH}_{2}\right), 2.11-2.24\left(\mathrm{~m}, 2 \mathrm{H}, \mathrm{CH}_{2}\right)$, 3.82 - $3.95\left(\mathrm{~m}, 1 \mathrm{H}, \mathrm{HOCHCHN}^{1}\right), 4.34-4.57\left(\mathrm{~m}, 2 \mathrm{H},{ }^{+} \mathrm{N}^{4} \mathrm{CH}_{2}\right), 4.66-4.75(\mathrm{~m}, 1 \mathrm{H}$, $\left.\mathrm{HOCHCHN}^{1}\right), 8.52\left(\mathrm{~s}, 1 \mathrm{H}, \mathrm{CHN}^{1} \mathrm{~N}^{2}=\mathrm{CHN}^{4}\right), 10.73\left(\mathrm{~s}, 1 \mathrm{H}, \mathrm{CHN}^{1} \mathrm{CH}^{4} \mathrm{~N}^{4}\right) ;{ }^{13} \mathrm{C} \mathrm{NMR}\left(\mathrm{CDCl}_{3}\right.$, $100 \mathrm{MHz}) \delta$ : $14.06\left(\mathrm{CH}_{2} \mathrm{CH}_{3}\right), 22.60\left(\mathrm{CyCH}_{2}\right), 23.96\left({ }^{+} \mathrm{N}^{4} \mathrm{CH}_{2} \mathrm{CH}_{2}\right), 24.10\left(\mathrm{CH}_{2} \mathrm{CH}_{3}\right), 26.29$ $\left(\mathrm{CyCH}_{2}\right), \quad 28.90 \quad\left({ }^{+} \mathrm{N}^{4} \mathrm{CH}_{2} \mathrm{CH}_{2} \mathrm{CH}_{2}\right), \quad 29.19 \quad\left({ }^{+} \mathrm{N}^{4} \mathrm{CH}_{2} \mathrm{CH}_{2} \mathrm{CH}_{2} \mathrm{CH}_{2} \mathrm{CH}_{2}\right), \quad 29.34$ $\left({ }^{+} \mathrm{N}^{4} \mathrm{CH}_{2} \mathrm{CH}_{2} \mathrm{CH}_{2} \mathrm{CH}_{2}\right), 29.40\left(\mathrm{CyCH}_{2}\right), 29.52\left(\mathrm{CH}_{2} \mathrm{CH}_{2} \mathrm{CH}_{2} \mathrm{CH}_{3}\right), 29.61\left(\mathrm{CH}_{2} \mathrm{CH}_{2} \mathrm{CH}_{2} \mathrm{CH}_{2} \mathrm{CH}_{3}\right)$, $31.79 \quad\left(\mathrm{CH}_{2} \mathrm{CH}_{2} \mathrm{CH}_{3}\right), \quad 33.78 \quad\left(\mathrm{CyCH}_{2}\right), \quad 49.05 \quad\left({ }^{+} \mathrm{N}^{4} \mathrm{CH}_{2}\right), \quad 68.55 \quad\left(\mathrm{HOCHCHN}^{1}\right), \quad 70.96$ $\left(\mathrm{HOCHCHN}{ }^{1}\right), 142.30\left(\mathrm{CHN}^{1} \mathrm{~N}^{2}=C \mathrm{HN}^{4}\right), 143.06\left(\mathrm{CHN}^{1} \mathrm{CH}=\mathrm{N}^{4}\right)$; Anal. Calcd. (\%) for $\mathrm{C}_{18} \mathrm{H}_{34} \mathrm{IN}_{3} \mathrm{O}: \mathrm{C}, 49.66 \mathrm{H}, 7.87 \mathrm{~N}, 9.65$. Found: C, $49.65 \mathrm{H}, 7.82 \mathrm{~N}, 9.61 ;[\alpha]_{\mathrm{D}}{ }^{26}+7.8(\mathrm{c}=1.5$, $\mathrm{CHCl}_{3}$ ) for ee $99 \%$. 


\section{Procedures for anions exchange in triazolium ionic liquids}

Lead(II) trifluoroacetate. To the solution of a lead oxide $(1 \mathrm{~g})$ in $5 \mathrm{ml}$ of distilled $\mathrm{H}_{2} \mathrm{O}$, stirred at $0{ }^{\circ} \mathrm{C}, 50 \%$ water solution of $\mathrm{CF}_{3} \mathrm{COOH}$ was added dropwise until $\mathrm{pH}$ 6-6.5 was reached. Pink suspension was filtered off and the permeate was partially condensed on rotary evaporator to give colorless liquid ready to use.

\section{General procedure for anion exchange (7a-7d)}

To the stirred solution of the appropriate bromide salt $(0.1 \mathrm{~g})$ in distilled water $(3 \mathrm{ml})$, $\left(\mathrm{CF}_{3} \mathrm{COO}\right)_{2} \mathrm{~Pb}$ was added drop by drop at $0{ }^{\circ} \mathrm{C}$ till all bromide anions became precipitated. Then the reaction suspension was filtrated by using Pasteur pipette tipped with wool. To a sustain permeate toluene $(15 \mathrm{ml})$ was added in order to remove the last trace of water azeotropicly. This procedure was repeated 5-times and then the solid residue was rinsed with diethyl ether $(3 \times 3$ $\mathrm{ml})$. The collected washings were evaporated under reduced pressure yielding brown semitransparent oil. The oil was diluted with acetone $(2 \mathrm{ml})$ and filtered through active carbon. The product was evaporated to dryness to give transparent colorless oil.

4-Ethyl-1-[(1S,2S)-2-hydroxycyclohexyl]-1H-1,2,4-triazol-4-ium trifluoroacetate (7a). Colourless oil; yield 96\%; $\mathrm{R}_{f} 0.13\left[\mathrm{CHCl}_{3}-\mathrm{MeOH}(8: 2)\right] ;{ }^{1} \mathrm{H} \mathrm{NMR}\left(\mathrm{CDCl}_{3}, 400 \mathrm{MHz}\right) \delta: 1.28$ $1.54\left(\mathrm{~m}, 4 \mathrm{H}, \mathrm{CH}_{2}\right), 1.58\left(\mathrm{t}, J=7.28 \mathrm{~Hz}, 3 \mathrm{H}, \mathrm{CH}_{2}\right), 1.77-2.27\left(\mathrm{~m}, 5 \mathrm{H}, \mathrm{CH}_{2}\right), 3.76(\mathrm{~s}, 1 \mathrm{H}$, $\left.\mathrm{HOCHCHN}^{1}\right), 4.31-4.58\left(\mathrm{~m}, 3 \mathrm{H}, \mathrm{HOCHCHN}{ }^{1}\right.$ and $\left.{ }^{+} \mathrm{N}^{4} \mathrm{CH}_{2} \mathrm{CH}_{3}\right), 8.28\left(\mathrm{~s}, 1 \mathrm{H}, \mathrm{CHN}^{1} \mathrm{~N}^{2}=\mathrm{CHN}^{4}\right)$, $10.49\left(\mathrm{~s}, 1 \mathrm{H}, \mathrm{CHN}^{1} \mathrm{CH}=\mathrm{N}^{4}\right) ;{ }^{13} \mathrm{C} \mathrm{NMR}\left(\mathrm{CDCl}_{3}, 100 \mathrm{MHz}\right) \delta: 14.73\left(\mathrm{CH}_{2} \mathrm{CH}_{3}\right), 23.95\left(\mathrm{CyCH}_{2}\right)$, $24.04\left(\mathrm{CyCH}_{2}\right), 29.71\left(\mathrm{CyCH}_{2}\right), 33.91\left(\mathrm{CyCH}_{2}\right), 43.74\left({ }^{+} \mathrm{N}^{4} \mathrm{CH}_{2} \mathrm{CH}_{3}\right), 68.48\left(\mathrm{HOCHCHN}^{1}\right)$, $71.37\left(\mathrm{HOCHCHN}^{1}\right), 127.79$ (q, $\left.1 \mathrm{C}, J 292 \mathrm{~Hz}, C \mathrm{~F}_{3}\right), 141.91\left(\mathrm{CHN}^{1} \mathrm{~N}^{2}=C \mathrm{HN}^{4}\right), 143.38$ $\left(\mathrm{CHN}^{1} \mathrm{CH}=\mathrm{N}^{4}\right), 162.23\left(\mathrm{q}, 1 \mathrm{C}, J 32.4 \mathrm{~Hz}, \mathrm{O}=C \mathrm{CF}_{3}\right)$; HRMS $\left(\mathrm{ESI}^{+}, \mathrm{m} / z\right):\left[\mathrm{M}^{+}\right]_{\text {calcd }}=196.1439$, $\left[\mathrm{M}^{+}\right]_{\text {found }}=196.0939,\left(\mathrm{ESI}^{-}, \mathrm{m} / \mathrm{z}\right):\left[\mathrm{M}^{-}\right]_{\text {calcd }}=112.9850,\left[\mathrm{M}^{-}\right]_{\text {found }}=112.9035 ;$ Anal. Calcd.$(\%)$ for $\mathrm{C}_{12} \mathrm{H}_{18} \mathrm{~F}_{3} \mathrm{~N}_{3} \mathrm{O}_{3}$ : C, $46.60 \mathrm{H}, 5.87 \mathrm{~N}, 13.59$. Found: $\mathrm{C}, 46.57 \mathrm{H}, 5.51 \mathrm{~N}, 13.54 ;[\alpha]_{\mathrm{D}}{ }^{23.5}+6.5(\mathrm{c}=$ $1.07, \mathrm{CHCl}_{3}$ ) for ee $99 \%$.

4-Propyl-1-[(1S,2S)-2-hydroxycyclohexyl]-1H-1,2,4-triazol-4-ium trifluoroacetate (7b). Colorless oil; yield 98\%; $\mathrm{R}_{f} 0.11\left[\mathrm{CHCl}_{3}-\mathrm{MeOH}(8: 2)\right] ;{ }^{1} \mathrm{H} \mathrm{NMR}\left(\mathrm{CDCl}_{3}, 400 \mathrm{MHz}\right) \delta$ : 0.99 (t, $J$ $\left.=7.33 \mathrm{~Hz}, 3 \mathrm{H}, \mathrm{CH}_{2} \mathrm{CH}_{3}\right), 1.32-1.59\left(\mathrm{~m}, 4 \mathrm{H}, \mathrm{CH}_{2}\right), 1.79-2.02\left(\mathrm{~m}, 6 \mathrm{H}, \mathrm{CH}_{2}\right), 2.19(\mathrm{~s}, 1 \mathrm{H}, \mathrm{OH})$, 3.68 - $3.79\left(\mathrm{~m}, 1 \mathrm{H}, \mathrm{HOCHCHN}{ }^{1}\right), 4.20$ - $4.41\left(\mathrm{~m}, 2 \mathrm{H}, \mathrm{N}^{4} \mathrm{CH}_{2} \mathrm{CH}_{2} \mathrm{CH}_{3}\right), 4.47$ - $4.57(\mathrm{~m}, 1 \mathrm{H}$,

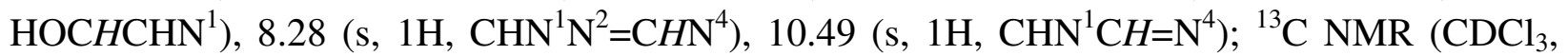
$100 \mathrm{MHz}) \delta$ : $10.41\left(\mathrm{CH}_{2} \mathrm{CH}_{3}\right), 23.20\left(\mathrm{CH}_{2} \mathrm{CH}_{3}\right), 23.97\left(\mathrm{CyCH}_{2}\right), 24.01\left(\mathrm{CyCH}_{2}\right), 29.61$ $\left(\mathrm{CyCH}_{2}\right), 33.86(\mathrm{CyCH}), 49.82\left({ }^{+} \mathrm{N}^{4} \mathrm{CH}_{2} \mathrm{CH}_{2} \mathrm{CH}_{3}\right), 68.45\left(\mathrm{HOCHCHN}^{1}\right), 71.43\left(\mathrm{HOCHCHN}^{1}\right)$, $116.84-120.25\left(\mathrm{q}, J 292 \mathrm{~Hz}, C F_{3}\right), 142.38\left(\mathrm{CHN}^{1} \mathrm{~N}^{2}=C \mathrm{HN}^{4}\right), 143.51\left(\mathrm{CHN}^{1} C \mathrm{H}^{2} \mathrm{~N}^{4}\right), 162.17$ $162.99\left(\mathrm{q}, J=32.4 \mathrm{~Hz}, \mathrm{O}=C C_{3}\right)$; HRMS $\left(\mathrm{ESI}^{+}, \mathrm{m} / \mathrm{z}\right):\left[\mathrm{M}^{+}\right]_{\text {calcd }}=210.1595,\left[\mathrm{M}^{+}\right]_{\text {found }}=$ 210.0975, $\left(\mathrm{ESI}^{-}, \mathrm{m} / z\right):\left[\mathrm{M}^{-}\right]_{\text {calcd }}=112.9850,\left[\mathrm{M}^{-}\right]_{\text {found }}=112.9035$; Anal. Calcd. $(\%)$ for $\mathrm{C}_{13} \mathrm{H}_{20} \mathrm{~F}_{3} \mathrm{~N}_{3} \mathrm{O}_{3}$ : C, $48.29 \mathrm{H}, 6.24 \mathrm{~N}, 13.00$. Found: $\mathrm{C}, 48.25 \mathrm{H}, 6.18 \mathrm{~N}, 12.92 ;[\alpha]_{\mathrm{D}}^{23.5}+2.3(\mathrm{c}=$ $1.3, \mathrm{CHCl}_{3}$ ) for ee $99 \%$.

4-(Prop-2-en-1-yl)-1-[(1S,2S)-2-hydroxycyclohexyl]-1H-1,2,4-triazol-4-ium trifluoroacetate (7c). Colorless oil; yield 94\%; $\mathrm{R}_{f} 0.09\left[\mathrm{CHCl}_{3}-\mathrm{MeOH}(8: 2)\right] ;{ }^{1} \mathrm{H} \mathrm{NMR}\left(\mathrm{CDCl}_{3}, 400 \mathrm{MHz}\right) \delta$ : 
1.13 - $2.24\left(\mathrm{~m}, 8 \mathrm{H}, \mathrm{CyCH}_{2}\right), 2.81(\mathrm{~s}, 1 \mathrm{H}, \mathrm{OH}), 3.72-3.78\left(\mathrm{~m}, 1 \mathrm{H}, \mathrm{HOCHCHN}^{1}\right), 4.44-4.53$ $\left(\mathrm{m}, 1 \mathrm{H}, \mathrm{HOCHCHN}^{1}\right), 4.97\left(\mathrm{dd}, J 15.12,6.51 \mathrm{~Hz}, 2 \mathrm{H},{ }^{+} \mathrm{NCH}_{2} \mathrm{CH}_{\mathrm{a}}\right), 5.29-5.57(\mathrm{~m}, 2 \mathrm{H}$, $\left.{ }^{+} \mathrm{NCH}_{2} \mathrm{CH}_{\mathrm{a}}=\mathrm{CH}_{\mathrm{a}} \mathrm{H}_{\mathrm{b}}\right), 5.92-6.05\left(\mathrm{~m}, 1 \mathrm{H},{ }^{+} \mathrm{NCH}_{2} \mathrm{CH}_{\mathrm{a}}=\mathrm{CH}_{\mathrm{a}} \mathrm{H}_{\mathrm{b}}\right), 8.24\left(\mathrm{~s}, 1 \mathrm{H}, \mathrm{CHN}^{1} \mathrm{~N}^{2}=\mathrm{CHN}^{4}\right)$, $10.27\left(\mathrm{~s}, 1 \mathrm{H}, \mathrm{CHN}^{1} \mathrm{CH}=\mathrm{N}^{4}\right) ;{ }^{13} \mathrm{C} \mathrm{NMR}\left(\mathrm{CDCl}_{3}, 100 \mathrm{MHz}\right) \delta: 23.88\left(\mathrm{CyCH}_{2}\right), 23.96\left(\mathrm{CyCH}_{2}\right)$, $29.63\left(\mathrm{CyCH}_{2}\right), 33.77\left(\mathrm{CyCH}_{2}\right), 50.53\left({ }^{+} \mathrm{NCH}_{2} \mathrm{CH}_{\mathrm{a}}\right), 68.37\left(\mathrm{HOCHCHN}^{1}\right), 71.50\left(\mathrm{HOCHCHN}^{1}\right)$, $120.33\left(\mathrm{q}, J 292 \mathrm{~Hz}, C \mathrm{~F}_{3}\right), 123.57\left({ }^{+} \mathrm{NCH}_{2} \mathrm{CH}_{\mathrm{a}}=\mathrm{CH}_{\mathrm{a}} \mathrm{H}_{\mathrm{b}}\right), 128.67\left({ }^{+} \mathrm{NCH}_{2} \mathrm{CH}_{\mathrm{a}}=C_{\mathrm{a}} \mathrm{H}_{\mathrm{b}}\right), 142.16$ $\left(\mathrm{CHN}^{1} \mathrm{~N}^{2}=C \mathrm{HN}^{4}\right), 143.33\left(\mathrm{CHN}^{1} C H=\mathrm{N}^{4}\right), 163.01\left(\mathrm{q}, J 32.4 \mathrm{~Hz}, \mathrm{O}=C \mathrm{C} F_{3}\right) ; \mathrm{HRMS}\left(\mathrm{ESI}^{+}, m / z\right)$ : $\left[\mathrm{M}^{+}\right]_{\text {calcd }}=208.1439,\left[\mathrm{M}^{+}\right]_{\text {found }}=208.0848,\left(\mathrm{ESI}^{-}, \mathrm{m} / \mathrm{z}\right):\left[\mathrm{M}^{-}\right]_{\text {calcd }}=112.9850,\left[\mathrm{M}^{-}\right]_{\text {found }}=$ 112.9035; Anal. Calcd. (\%) for $\mathrm{C}_{13} \mathrm{H}_{18} \mathrm{~F}_{3} \mathrm{~N}_{3} \mathrm{O}_{3}$ : C, $48.60 \mathrm{H}, 5.65 \mathrm{~N}, 13.08$. Found: C, $48.55 \mathrm{H}$, $5.63 \mathrm{~N}, 13.04 ;[\alpha]_{\mathrm{D}}{ }^{23.5}+4.8\left(\mathrm{c}=1.03, \mathrm{CHCl}_{3}\right)$ for ee $99 \%$.

4-Butyl-1-[(1S,2S)-2-hydroxycyclohexyl]-1H-1,2,4-triazol-4-ium trifluoroacetate (7d). Colourless oil; yield 99\%; $\mathrm{R}_{f} 0.1\left[\mathrm{CHCl}_{3}-\mathrm{MeOH}(8: 2)\right] ;{ }^{1} \mathrm{H} \mathrm{NMR}\left(\mathrm{CDCl}_{3}, 400 \mathrm{MHz}\right) \delta: 0.94$ (t, $J$ $\left.=7.21 \mathrm{~Hz}, 3 \mathrm{H}, \mathrm{CH}_{2} \mathrm{CH}_{3}\right), 1.09-1.59\left(\mathrm{~m}, 6 \mathrm{H}, \mathrm{CH}_{2}\right), 1.63-2.03\left(\mathrm{~m}, 5 \mathrm{H}, \mathrm{CH}_{2}\right), 2.10-2.15(\mathrm{~m}$, $\left.2 \mathrm{H},{ }^{+} \mathrm{N}^{4} \mathrm{CH}_{2}\right), 3.49$ - $3.92\left(\mathrm{~m}, 1 \mathrm{H}, \mathrm{HOCHCHN^{1 }}\right), 4.07$ - $4.64\left(\mathrm{~m}, 3 \mathrm{H}, \mathrm{HOCHCHN}^{1}\right), 8.38(\mathrm{~s}, 1 \mathrm{H}$,

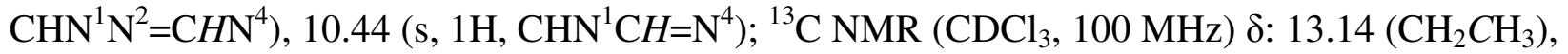
$19.27\left(\mathrm{CH}_{2} \mathrm{CH}_{3}\right), 23.97\left(\mathrm{CyCH}_{2}\right), 24.03\left(\mathrm{CyCH}_{2}\right), 29.68\left(\mathrm{CH}_{2} \mathrm{CH}_{2} \mathrm{CH}_{3}\right), 31.57\left(\mathrm{CyCH}_{2}\right), 33.83$ $\left(\mathrm{CyCH}_{2}\right), 48.10\left({ }^{+} \mathrm{N}^{4} \mathrm{CH}_{2} \mathrm{CH}_{2} \mathrm{CH}_{2} \mathrm{CH}_{3}\right), 68.45\left(\mathrm{HOCHCHN}^{1}\right), 71.40\left(\mathrm{HOCHCHN}^{1}\right), 127.52(\mathrm{q}, 1$ C, J $292 \mathrm{~Hz},), 142.78\left(\mathrm{CHN}^{1} \mathrm{~N}^{2}=C \mathrm{HN}^{4}\right), 143.23\left(\mathrm{CHN}^{1} C \mathrm{H}^{4} \mathrm{~N}^{4}\right), 163.25(\mathrm{q}, 1 \mathrm{C}, J 32.4 \mathrm{~Hz}$,$) ;$ $\operatorname{HRMS}\left(\mathrm{ESI}^{+}, \mathrm{m} / \mathrm{z}\right):\left[\mathrm{M}^{+}\right]_{\text {calcd }}=224.1752,\left[\mathrm{M}^{+}\right]_{\text {found }}=224.1096,\left(\mathrm{ESI}^{-}, \mathrm{m} / \mathrm{z}\right):\left[\mathrm{M}^{-}\right]_{\text {calcd }}=112.9850$, $\left[\mathrm{M}^{-}\right]_{\text {found }}=112.9035$; Anal. Calcd. $(\%)$ for $\mathrm{C}_{14} \mathrm{H}_{22} \mathrm{~F}_{3} \mathrm{~N}_{3} \mathrm{O}_{3}: \mathrm{C}, 49.85 \mathrm{H}, 6.57 \mathrm{~N}, 12.46$. Found: $\mathrm{C}$, $49.81 \mathrm{H}, 6.53 \mathrm{~N}, 12.44 ;[\alpha]_{\mathrm{D}}^{23.5}+1.6\left(\mathrm{c}=1.27, \mathrm{CHCl}_{3}\right)$ for ee $99 \%$.

\section{Acknowledgements}

The project was co-financed by The European Regional Development Fund under The Innovative Economy Operational Programme 2007-2013: "Biotransformations for Pharmaceutical and Cosmetic Industry" POIG.01.03.01-00-158/09. These studies were partially supported by Warsaw University of Technology, Faculty of Chemistry. In addition, we would like to thank Prof. Ludwik Synoradzki and Krzysztof Bujnowski, PhD. for providing assistance with laboratory space and equipment.

\section{References}

1. Borowiecki, P. M.Sc. Thesis, Warsaw University of Technology, Warsaw 2010.

2. (a) Welton, T. Chem. Rev. 1999, 99, 2071-2083; (b) Wasserscheid, P.; Welton, T. Ionic Liquids in Synthesis; Wiley-VCH: Weinheim, Germany, 2003. (c) Wasserscheid, P.; Keim, W. Angew. Chem., Int. Ed. 2000, 39, 3772-3789. (d) Dupont, J.; de Souza, R.F.; Suarez P. A. 
Z. Chem. Rev. 2002, 102, 3667-3692. (e) Welton, T. Coord. Chem. Rev. 2004; 248, 24592477.

3. Wasserscheid, P.; Welton, T. Ionic Liquids in Synthesis; Wiley-VCH: Weinheim, Germany, 2003.

4. Jain, N.; Kumar, A.; Chauhan, S.; Chauhan, S. M. S. Tetrahedron 2005, 61, 1015-1060.

5. (a) Baudequin, C.; Brégeon, D.; Levillain, J.; Guillen, F.; Plaquevent, J.-C.; Gaumont, A.-C. Tetrahedron: Asymmetry 2005, 16, 3921-3945; (b) Vallette, H.; Ferron, L.; Coquerel, G.; Guillen, F.; Plaqueventa, J.-C. Arkivoc 2006, (iv), 200-211.

6. Ni, B.; Zhang, Q.; Headley, A. D. Tetrahedron Lett. 2008, 49, 1249-1252.

7. (a) Shah, S.; Gupta, M. N. Bioorg. Med. Chem. Lett. 2007, 17, 921-924. (b) Laszlo, J. A.; Compton, D. Biotechnol. Bioeng. 2001, 75, 181-186.

8. (a) Zhu. H-P; Yang, F.; Tang. J. Green Chem. 2003, 5, 38-39. (b) Wang, Z.;Wang, Q.; Zhang, Y.; Bao,W. Tetrahedron Lett. 2005, 46, 4657-4660. (c) Ni, B.; Zhang, Q.; Headley, A. D. Green Chem. 2007, 9, 737-739. (d) Zhou,W.; Xu, L.W.; Qiu, H. Y.; Lai, G. Q.; Xia, C. G.; Jiang, J. X. Helv. Chim. Acta 2008, 91, 53-59.

9. (a) Pegot, B.; Vo-Thanh, G.; Gori, D. Tetrahedron Lett. 2004, 45, 6425-6428. (b) Baudequin, C.; Brégeon, D.; Levillain, J.; Guillen, F.; Plaquevent, J.-C.; Gaumont, A.-C. Tetrahedron: Asymmetry 2005, 16, 3921. (c) Sanzhong Luo; Xueling Mi; Long Zhang Angew. Chemie, Int. Ed. 2006, 45, 3093-3097. (d) Ni, B.; Zhang, Q.; Headley, A. D. Tetrahedron Lett. 2008, 49, 1249-1252.

10. (a) Biedroń, T.; Kubisa, P. Polym. Int. 2003, 52, 1584-1588. (b) Ma, H.-Y.; Wan, X.-H.; Chen, X.-F.; Zhou, Q. F. Chin. J. Polym. Sci. 2003, 21, 265-270. (c) Biedroń, T.; Kubisa, P. J. Polym. Sci., Part A: Polym. Chem. 2005, 43, 3454-3459.

11. (a) Ding, J.; Welton, T.; Armstrong, D. W. Anal. Chem. 2004, 76, 6819-6822. (b) Rizvi, S. A. A.; Shamsi, S. A. Anal. Chem. 2006, 78, 7061-7069.

12. (a) Wasserscheid, P.; Bosmann, A.; Bolm, C. Chem. Commun. 2002, 3, 200-201. (b) Ishida, Y.; Miyauchi, H.; Saigo, K. Chem. Commun. 2002, 19, 2240-2241. (c) Levillain, J.; Dubant, G.; Abrunhosa, I.; Gulea, M.; Gaumont, A.-C. Chem. Commun. 2003, 23, 2914-2915. (d) Clavier, H.; Boulanger, L.; Audic, N.; Toupet, L.; Mauduit, M.; Guillemin, J.-C. Chem. Commun. 2004, 10, 1224-1225. (e) Ishida, Y.; Sasaki, D.; Miyauchi, H.; Saigo, K. Tetrahedron Lett. 2004, 45, 9455-9459.

13. (a) Tosoni, M.; Laschat, S.; Baro, A. Helv. Chim. Acta 2004, 87, 2742-2749. (b) Baudoux, J.; Judeinstein, P.; Cahard, D.; Plaquevent, J.-C. Tetrahedron Lett. 2005, 46, 1137-1140.

14. Łuczak, J.; Jungnickel, C.; Łącka, I.; Stolte, S.; Hupka, J. Green Chemistry 2010, 12, 593601.

15. Pernak, A.; Majewski, P.; Iwanik, K.; Grzymisławski, M.; Pernak, J. Acta Histochemica 2003, 105, 135-142. (b) Pernak, J.; Syguda, A.; Mirska, I.; Pernak, A. Nawrot, J. Prądzyńska, A.; Griffin, S. T.; Rogers, R. D.; Chem. Eur. J. 2007, 13, 6817-6827.

16. (a) Wu, Y. S.; Lee, H. K.; Li, F. Y. Journal of Chromatography A 2001, 912, 171-179. (b) Benson, F. R. Tetrazoles, Tetrazines and Purines and Related Ring System, Heterocyclic 
Compounds, Elderfield, R. C., Ed. Wiley: New York, 1967; Vol. 8, pp 1-104. (c) Demirayak, S.; Benkli, K.; Guven, K. Pharm. Acta Helv. 1998, 72, 285-290. (d) Tanoury, G. J.; Senanayake, C. H.; Hett, R.; Kuhn, A. M.; Kessler, D. W.; Wald, S. A. Tetrahedron Lett. 1998, 39, 6845-6848.

17. Rios-Lombard, N.R.; Porcar, R.; Busto, E.; Alfonso, I.; Montejo-Bernardo, J.; GarciaGranda, S.; Gotor, V.; Luis, S. V.; Garcia-Verdugo, E.; Gotor-Fernández, V. Chem. Cat. Chem 2011, 3, 1921-1928.

18. Torregrosa, R.; Pastor, I. M.; Yus, M. Tetrahedron 2007, 63, 469-473.

19. Busto, E.; Gotor-Fernandez, V.; Rios-Lombardia, N.; Garcia-Verdugo, E. A. I.; GarciaGranda, S.; Menendez-Velazquez, A.; Burguete, M. I.; Luis, S. V.; Gotor, V. Tetrahedron Letters 2007, 48; 5251-5254.

20. Dale, J. A.; Mosher, H. S. J. Am. Chem. Soc. 1973, 95, 512-519.

21. Latypov, Sh. K.; Seco, J. M.; Quinoa, E.; Riguera, R. J. Org. Chem. 1995, 60, 504-515.

22. Faber, K.: Biotransformations in Organic Chemistry, Springer Verlag, 1997, pp 90-91.

23. (a) Mirzaei, Y. R.; Twamley, B.; Shreeve, J. M. J. Org. Chem. 2002, 67, 9340-9345. (b) Xue, H.; Twamley, B.; Shreeve, J. M. J. Org. Chem. 2004, 69, 1397-1400. (c) Schmidt, M. W.; Gordon, M. S.; Boatz, J. A. J. Phys. Chem. A 2005, 109, 7285-7295. 